\title{
A dynamical approach to the legal and illegal logging of forestry population and conservation using taxation
}

\author{
Manisha Chaudhary ${ }^{1 *}$ (D) and Rachana Pathak ${ }^{2}$
}

\author{
"Correspondence: \\ manisha23june@gmail.com; \\ mc2306@iiitm.ac.in.com \\ ${ }^{1}$ Department of Applied Sciences, \\ Indian Institute of Information \\ Technology and Management, \\ Morena Link Road, Gwalior, Madhya \\ Pradesh 474015, India \\ Full list of author information is \\ available at the end of the article
}

\begin{abstract}
Over-harvesting of forestry resource, primarily trees, through illegal logging has been exceptionally regular for decades. In the context of this worldwide issue, a mathematical model considering the joined impact of legal and illegal logging of trees from forestry biomass using delay-driven ordinary differential equations is proposed. For the set of equations, we have taken immature, mature forestry biomass, and industrial densities as three state variables. Additionally, the effect of time-lag for the conversion of immature forestry biomass to mature forestry biomass is considered. System boundedness, feasible equilibrium analysis and the stability of all the feasible equilibria is examined using the differential equation theory. From the detailed analysis of the system, it is observed that with the delay in time, the system bifurcates as it reaches the critical threshold. While without the delay, the system is asymptotically stable. Biologic and bio-economic results of the system are also interpreted for the optimal equilibrium solutions. The optimal path is obtained by constructing the Hamiltonian, which is further solved using Pontryagin"s principle associated with the control problem. Further, numerical simulation is also provided in support of analytical results. Moreover, the normalized forward sensitivity index is used to analyze the parameter sensitivity.
\end{abstract}

MSC: $34 \mathrm{D} ; 34 \mathrm{H} ; 90 \mathrm{~A} ; 92 \mathrm{~B}$

Keywords: delay system; Hopf bifurcation; optimal control; sensitivity analysis

\section{Introduction}

The forestry ecosystems are biologically richest on earth, exert the key influence on the biological diversity, human habitat, and the environmental conditions. Forestry biomass conserves landscapes, soil and water resources and provides services and products for the livelihood of millions of the living animals as well as humans. In many developing countries forests contribute towards the local employment, processing, and trade of forest products [1]. As per the examination on mangrove forest dynamics of the Sundarbans in Bangladesh and India, the timberland is the center for economic activities, such as the extraction of timber and fuel wood, fishing and collecting honey and other backwoodsbased items. About 300,000 individuals work regularly as woodcutters, palm collectors, angler, and honey hunters.

(c) The Author(s) 2017. This article is distributed under the terms of the Creative Commons Attribution 4.0 International License (http://creativecommons.org/licenses/by/4.0/), which permits unrestricted use, distribution, and reproduction in any medium, provided you give appropriate credit to the original author(s) and the source, provide a link to the Creative Commons license, and indicate if changes were made. 
Illegal logging and deforestation of forest land continue all over the world at an alarming rate. According to the report [2], an area of forests the size of Greece is lost every year. Now the time has come when the international community takes some protective action for the conservation of forestry population and legal logging. Also, the forest management committee should focus on increasing investment in sustainable forest management. Since illegal logging is significantly more profitable for industries than legal logging, the forest biomass is more rapidly decaying than the actual prediction. Chatham house in their briefing paper [3] saw significant effects as 17 million hectares of forest land were assessed to be protected from degradation. They likewise detailed that if the trees were legally logged, this could get USD 6.5 billion in extra income to the nations concerned. Also in this paper, they mentioned that more than half of the loss of forest in tropical countries is due to illegal logging and lack of social control. Areas affected by logging should be reforested with indigenous tree species. A worldwide program on the importance and ecological role of forests on biological diversity should be started by the government in schools and communities.

Previously, a lot of work has been done by researchers considering pollution, population, toxicant, industrialization, climate change (due to anthropogenic and natural causes) [48]. A palm oil plantation problem was undertaken for a nonlinear dynamical system [9]. The authors also obtained a control problem to estimate the control variables while maintaining the biomass level and maximize the oil palm production in a long period. In [10], the author discussed the competing effect of wood and synthetic-based industries using the ODE system. The author of [11] further modified the work considering the delay effect on the mature forestry biomass taking the DDE system. In [12], a mathematical model for the forestry biomass and the alternatives using the modified Leslie-Gower term was proposed. From the analysis, they observed that with increasing effort there is a decrease in both the mature forestry biomass and industrialization. Sufficient conditions for both persistence and global attraction of the system were obtained. Analytic results for the optimal harvesting policy and bionomic equilibrium were also presented in the analysis.

Forestry ecosystem and human habitat both are interrelated, and it attracts a lot of researchers, scientist as well as government bodies to study and understand the seriousness of the problem.

The dynamic growth model developed is based on the age-structure delay differential equation approach and is similar to that of Chaudhary et al. [10,11]. In this paper, an attempt is made to develop a dynamical model based on legal and illegal logging of forestry biomass for the set-up of forest-based industries. We consider taxation as a control instrument for logging since a large portion of forests are illegally logged and this does not come under the taxation. The paper is divided into several sections. An introduction is based on survey articles, reports, and the tools and techniques used in the present work. System dynamics and the assumption made for the set-up of the mathematical model are discussed in Section 2. Stability and system behavior in the presence of Hopf bifurcation, the direction of Hopf bifurcation, the control strategy used for the optimal use of forestry biomass, numerical simulation, and quantitative analysis are discussed in the later Sections 3, 4, 5, 6,7 , and 8 . Sensitivity analysis of the parameters associated with the coexistence state is discussed in Section 9. Finally, we conclude the work with salient result and discussion. 

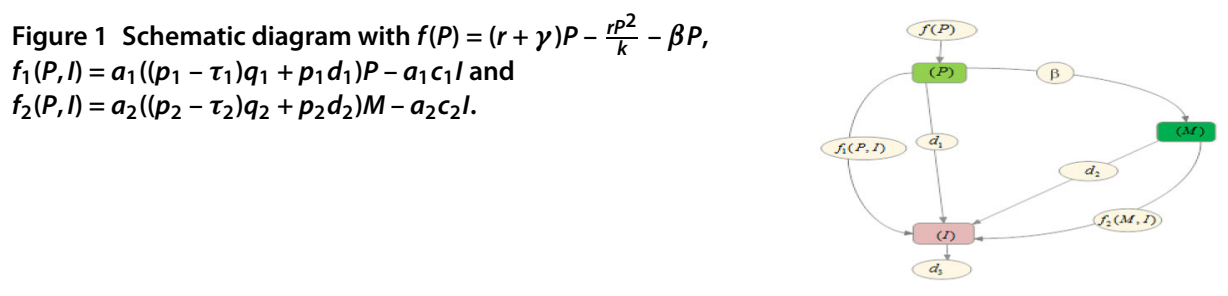

\section{System dynamics}

\subsection{Model assumptions}

Extending the work of [13], for the proposed system, we consider $P$ and $M$ as the immature and mature forestry biomass and $I$ as the industrial density which is directly proportional to the industrial efforts to harvest forestry biomass (both immature and mature). In the present study, we assume $h_{1}$ to be the maturation delay (time delayed to convert from immature to mature population). We also assume that there is illegal logging of forestry biomass done by industries for its growth, which is free from tax. This particular portion of illegal logging is defined by $d_{1}$ and $d_{2}$ for the immature and mature forestry biomass, respectively. Since a portion of forestry biomass is illegally cut down for the individual benefit, it is interesting to check how it affects both the immature and mature forestry biomass and industrial growth.

\subsection{Schematic flow}

By assumptions considered for the model definition, the schematic diagram is described in Figure 1.

\section{A delay-driven conceptual system}

Forest management planning relies on the outcome or the estimation of mathematical models using dynamical system theory and provides a framework for a flexible representation of varying disturbances in nature's biological diversity and human habitat.

For the system dynamics, we have considered three state variables: two of them are related to forestry biomass via immature and mature forestry biomass and the third is the industries which harvest both immature and mature forestry biomass. According to [14], in all the natural phenomena time lag or delay is so often that to ignore it is to ignore the reality. Here, for the present system, we consider $h_{1}$ as the delay parameter, or maturation delay, which is the actual time taken to convert an immature tree to a mature one. Also, for the third equation, it is considered that the industrial density is directly proportional to the industrial effort $E$, i.e., $I \propto E$, and hence we could write the rate of change in the industrial density as $\frac{d I}{d t}=\alpha \frac{d E}{d t}$. For simplicity, we take $\alpha=1$. The proposed model is governed by the following system of delay differential equations:

$$
\begin{aligned}
& \frac{d P}{d t}=(r+\gamma) P-\frac{r P^{2}}{K}-\beta P\left(t-h_{1}\right)-q_{1} P I-d_{1} P I, \\
& \frac{d M}{d t}=\beta P\left(t-h_{1}\right)-q_{2} M I-d_{2} M I-\mu M, \\
& \frac{d I}{d t}=\left[a_{1}\left(\left(p_{1}-\tau_{1}\right) q_{1}+p_{1} d_{1}\right) P+a_{2}\left(\left(p_{2}-\tau_{2}\right) q_{2}+p_{2} d_{2}\right) M-a_{1} c_{1}-a_{2} c_{2}-d_{3}\right] I .
\end{aligned}
$$


Table 1 Description of parameters

\begin{tabular}{lll}
\hline Parameter & Description & Unit \\
\hline$r$ & intrinsic growth rate & per yr \\
$\gamma$ & new plantation & per yr \\
$\mathrm{K}$ & carrying capacity & - \\
$\beta$ & transition rate & per yr \\
$q_{1}$ & harvesting rate comes under tax & per yr \\
$q_{2}$ & harvesting rate comes under tax & per yr \\
$d_{1}$ & depletion rate because of illegal cutting & per yr \\
$d_{2}$ & depletion rate because of illegal cutting & per yr \\
$d_{3}$ & depletion rate of industries & per yr \\
$\mu$ & depletion rate of mature trees for non-industrial uses & per yr \\
$a_{1}$ & measuring the strength of reaction of harvest effort & - \\
$a_{2}$ & measuring the strength of reaction of harvest effort & - \\
$p_{1}$ & price of forestry biomass & per unit \\
$p_{2}$ & price of forestry biomass & per unit \\
$c_{1}$ & harvesting cost of forestry biomass & per yr \\
$c_{2}$ & harvesting cost of forestry biomass & per yr \\
$\tau_{1}$ & tax of forestry biomass & per unit \\
$\tau_{2}$ & tax of forestry biomass & per unit \\
$h_{1}$ & delay parameter & years \\
\hline
\end{tabular}

Initially, $P(\varrho)=P_{0} \geq 0$ for $\varrho \epsilon\left[-h_{1}, 0\right], M(0) \geq 0, I(0) \geq 0$. The parameters chosen for the numerical simulation are hypothetical, and their description with corresponding units is given in Table 1.

\section{Positivity and system boundedness}

\subsection{Positivity of solutions}

For the positivity of all the solutions $P(t), M(t)$, and $I(t)$, the initial population is considered as $P(0)=P_{0}>0, M(0)=M_{0}>0$, and $I(0)=I_{0}>0$. From system equation (1), we have $\frac{d P}{d t} \geq$ $-\left(\beta P+q_{1} P I_{u}+d_{1} I_{u} M\right)$ on solving the inequality

$$
P \geq P_{0} \exp ^{-\left(\beta+q_{1} I_{u}+d_{1} I_{u}\right) t}
$$

Again, from equation (2), $\frac{d M}{d t} \geq-\left(\mu+q_{2} I_{u}+d_{2} I_{u}\right) M$ on solving the inequality

$$
M \geq M_{0} \exp ^{-\left(\mu+q_{2} I_{u}+d_{2} I_{u}\right) t}
$$

Similarly, from equation (3), $\frac{d I}{d t} \geq-\left(a_{1} c_{1}+a_{2} c_{2}+d_{3}\right) I$ on solving the inequality, we can write

$$
I \geq I_{0} \exp ^{-\left(a_{1} c_{1}+a_{2} c_{2}+d_{3}\right) t}
$$

Hence $P, M$, and $I$ are greater than zero for all $t \geq 0$. The value of $I_{u}$ is defined in the next Section 4.2. To analyze the model, we need the bounds of dependent variables involved. For this, we find the region of attraction in the following lemma. We show the boundedness of the system using a comparison theorem as discussed in [15].

\subsection{Boundedness of the system}

Lemma 1 With positive initial conditions, system (1)-(3) is uniformly bounded in the region $\chi=\left\{(P, M, I) \in R_{+}^{3}: 0 \leq P+M+I \leq \frac{\phi}{\eta}\right\}$, as $t \rightarrow \infty$. 
Proof From equation (1), we have $\frac{d P}{d t} \leq(r+\gamma) P-\frac{r P^{2}}{K}$. Now, using the comparison theorem as $t \rightarrow \infty$, we get $\lim _{t \rightarrow \infty} \sup P(t) \leq \frac{(r+\gamma) K}{r}=P_{u}$. Similarly, from equation (2), we have $\lim _{t \rightarrow \infty} \sup M(t) \leq \frac{\beta P_{u}}{\mu}=M_{u}$ as $t \rightarrow \infty$. For the whole system equations (1)-(3), let $W=P+M+I$, then

$$
\begin{aligned}
& \frac{d W}{d t} \leq(r+\gamma) P-\frac{r P^{2}}{k}-\mu M-\left(a_{1} c_{1}-a_{2} c_{2}+d_{3}\right) I, \\
& \text { or }=(r+\gamma+\psi) P-\frac{r P^{2}}{k}-\mu M-\left(a_{1} c_{1}-a_{2} c_{2}+d_{3} I\right), \\
& \text { or }=(r+\gamma+\psi) P_{u}-\eta W,
\end{aligned}
$$

where $\eta=\min \left\{\psi, \mu,\left(a_{1} c_{1}+a_{2} c_{2}+d_{3}\right)\right\}$. Now, after using the comparison theorem, we have $\lim _{t \rightarrow \infty} \sup W(t) \leq \frac{\phi}{\eta}$ as $t \rightarrow \infty$ or we could say some of $(P+M+I) \leq \frac{\phi}{\eta}$, i.e., $I_{u} \leq \frac{\phi}{\eta}$. This completes the proof of lemma.

\section{Equilibrium and stability analysis}

\subsection{Equilibrium analysis}

System (1)-(3) has three biologically feasible equilibrium points (positive equilibrium) that are described as follows:

Trivial equilibrium point $\bar{E}=(0,0,0)$ : Existence of the trivial case is obvious as all the three populations are extinct.

Axial equilibrium point $\tilde{E}=(\tilde{P}, \tilde{M}, 0): \tilde{P}=\frac{(K(r-\beta+\gamma))}{r}, \tilde{M}=\frac{(K \beta(r-\beta+\gamma))}{r \mu}$. Axial equilibrium exists when $(r+\gamma)>\beta$, i.e., the transition rate from immature to mature stage is less than the sum of intrinsic growth and government efforts. In this case industries no longer exist. Interior equilibrium point $\hat{E}=(\hat{P}, \hat{M}, \hat{I})$ : Existence of interior equilibrium depends upon the industrial density as both immature and mature forestry biomass are defined in terms of industrial density (I). Immature population $\hat{P}=\frac{K}{r}\left[(r+\gamma)-\left(q_{1}+d_{1}\right) \hat{I}-\beta\right]$, mature population $\hat{M}=\frac{\beta \hat{P}}{\left(q_{2}+d_{2}\right) \hat{I}+\mu}$, both $P$ and $M$ exist when $\hat{P}>0$, when $(r+\gamma)>\left(q_{1}+d_{1}\right) \hat{I}-\beta$ is satisfied, and $I$ is defined by the positive roots of the quadratic equation

$$
z_{1} \hat{I}^{2}+z_{2} \hat{I}+z_{3}=0
$$

The quadratic equation (7) has one positive root when $z_{3}$ is less than zero and is possible when $(r+\gamma)>\beta$. Values of $z_{1}, z_{2}$, and $z_{3}$ are given as $z_{1}=\left(a_{1} d_{1}^{2} d_{2} K+a_{1} d_{1}^{2} K q_{1}+a_{1} d_{1} d_{2} K q_{1}+\right.$ $\left.a_{1} d_{1} K q_{1}^{2}\right) p_{1}+\left(a_{1} d_{1} d_{2} K q_{1}+a_{1} d_{1} K q_{1}^{2}+a_{1} d_{2} K q_{1}^{2}+a_{1} K q_{1}^{3}\right)\left(p_{1}-\tau_{1}\right), z_{2}=\left(a_{1} d_{2} K q_{1} \beta+\right.$ $\left.a_{1} K q_{1}^{2} \beta-a_{1} d_{2} K q_{1} r-a_{1} K q_{1}^{2} r-a_{1} d_{2} K q_{1} \gamma-a_{1} K q_{1}^{2} \gamma+a_{1} d_{1} K q_{1} \mu+a_{1} K q_{1}^{2} \mu\right)\left(p_{1}-\tau_{1}\right)+$ $\left(a_{2} d_{1} K q_{2} \beta+a_{2} K q_{1} q_{2} \beta\right)\left(p_{2}-\tau_{2}\right)+\left(a_{1} d_{1} d_{2} K \beta+a_{1} d_{1} K q_{1} \beta-a_{1} d_{1} d_{2} K r-a_{1} d_{1} K q_{1} r-\right.$ $\left.a_{1} d_{1} d_{2} K \gamma-a_{1} d_{1} K q_{1} \gamma+a_{1} d_{1}^{2} K \mu+a_{1} d_{1} K q_{1} \mu\right) p_{1}+\left(a_{2} d_{1} K d_{2} \beta+a_{2} K d_{2} q_{1} \beta\right) p_{2}+a_{1} c_{1} d_{2} r+$ $a_{2} c_{2} d_{2} r+d_{2} d_{3} r+a_{1} c_{1} q_{1} r+a_{2} c_{2} q_{1} r+d_{3} q_{1} r$, and $z_{3}=\left(a_{2} K d_{2} \beta^{2}-a_{2} K d_{2} r \beta-a_{2} K d_{2} \beta \gamma\right) p_{2}+$ $\left(a_{1} d_{1} K \beta \mu-a_{1} d_{1} K r \mu-a_{1} d_{1} K \gamma \mu\right) p_{1}+\left(a_{1} K q_{1} \beta \mu-a_{1} K q_{1} r \mu-a_{1} K q_{1} \gamma \mu\right)\left(p_{1}-\tau_{1}\right)+$ $\left(a_{2} K q_{2} \beta^{2}-a_{2} K q_{2} r \beta-a_{2} K q_{2} \beta \gamma\right)\left(p_{2}-\tau_{2}\right)+a_{1} c_{1} r \mu+a_{2} c_{2} r \mu+d_{3} r \mu$.

In the next section, the local stability criteria are obtained for the co-existence state, as we are interested in the state, when all the populations coexist for the defined system. 


\subsection{Stability analysis}

\subsubsection{System without maturation delay}

In this section, we show the local stability of the system for all the feasible equilibria. A variational matrix corresponding to system (1)-(3) is given as follows:

$$
J=\left[\begin{array}{ccc}
j_{11} & 0 & j_{13} \\
j_{21} & j_{22} & j_{23} \\
j_{31} & j_{32} & j_{33}
\end{array}\right]
$$

where $j_{11}=r-\beta+\gamma-\frac{2 r P}{K}-\left(d_{1}+q_{1}\right) I, j_{13}=-\left(d_{1}+q_{1}\right) P, j_{21}=\beta, j_{22}=-\left(d_{2}+q_{2}\right) I-\mu$, $j_{23}=-\left(d_{2}+q_{2}\right) M, j_{31}=a_{1} I\left(d_{1} p_{1}+q_{1}\left(p_{1} \tau_{1}\right)\right), j_{32}=a_{2} I\left(d_{2} p_{2}+q_{2}\left(p_{2} \tau_{2}\right)\right)$, and $j_{33}=a_{1} P\left(d_{1} p_{1}+\right.$ $\left.q_{1}\left(p_{1} \tau_{1}\right)\right)+a_{2} M\left(d_{2} p_{2}+q_{2}\left(p_{2} \tau_{2}\right)\right)-a_{1} c_{1}-a_{2} c_{2}-d_{3}$. It is observed that:

1. Trivial equilibrium $\bar{E}$ is obvious and is unstable.

2. Axial equilibrium $\tilde{E}$ with eigenvalues $-\frac{r \tilde{P}}{K},-\mu$, and $-a_{1} c_{1}-a_{2} c_{2}-d_{3}+a_{1} d_{1} \tilde{P} p_{1}+a_{2} d_{2} \tilde{M} p_{2}+a_{1} \tilde{P}\left(p_{1}-\tau_{1}\right) q_{1}+a_{2} \tilde{M}\left(p_{2}-\tau_{2}\right) q_{2}$ is stable when $a_{1} c_{1}+a_{2} c_{2}+d_{3}>a_{1} d_{1} \tilde{P} p_{1}+a_{2} d_{2} \tilde{M} p_{2}+a_{1} \tilde{P}\left(p_{1}-\tau_{1}\right) q_{1}+a 2 \tilde{M}\left(p_{2}-\tau_{2}\right) q_{2}$.

3. The stability of interior equilibrium $\hat{E}$ is an interesting case as all the state variables, immature (P) and mature (M) forestry biomass together with industrialization (I) coexist; analysis of them is as follows.

A variational matrix for the interior equilibrium $\hat{E}$ is given by

$$
J_{1}=\left[\begin{array}{ccc}
-\frac{r \hat{P}}{K} & 0 & -\left(d_{1}+q_{1}\right) \hat{P} \\
\beta & -\mu & -\left(d_{2}+q_{2}\right) \hat{M} \\
a_{1} \hat{I}\left(d_{1} p_{1}+q_{1}\left(p_{1}-\tau_{1}\right)\right) & a_{2} \hat{I}\left(d_{2} p_{2}+q_{2}\left(p_{2}-\tau_{2}\right)\right) & 0
\end{array}\right],
$$

the corresponding characteristic equation for the variational matrix $J$ is

$$
\lambda^{3}+Z_{1} \lambda^{2}+Z_{2} \lambda+Z_{3}=0
$$

where $Z_{1}=d_{2} \hat{I}+q_{2} \hat{I}+\frac{r \hat{P}}{K}+\mu, Z_{2}=\frac{r \hat{P}}{K}\left(\left(d_{2}+q_{2}\right) \hat{I}+\mu\right)+\left(a_{1} d_{1}^{2} \hat{I} \hat{P}+a_{1} d_{1} \hat{I} \hat{P} q_{1}\right) p_{1}+$ $\left(a_{2} d_{2}^{2} \hat{I} \hat{M}+a_{2} d_{2} \hat{I} \hat{M} q_{2}\right) p_{2}+\left(a_{1} d_{1} \hat{I} \hat{P} q_{1}+a_{1} \hat{I} \hat{P} q_{1}^{2}\right)\left(p_{1}-\tau_{1}\right)+\left(a_{2} d_{2} \hat{I} \hat{M} q_{2}+a_{2} \hat{I} \hat{M} q_{2}^{2}\right)\left(p_{2}-\tau_{2}\right)$ and $Z_{3}=\left(a_{1} d_{1}^{2} d_{2} I^{2} \hat{P}+a_{1} d_{1} d_{2} \hat{I}^{2} P q_{1}+a_{1} d_{1}^{2} \hat{I}^{2} \hat{P} q_{2}+a_{1} d_{1} \hat{I}^{2} \hat{P} q_{1} q_{2}+a_{1} d_{1}^{2} \hat{I} \hat{P} \mu+a_{1} d_{1} \hat{I} \hat{P} q_{1} \mu\right) p_{1}+$ $\left(a_{1} d_{1} d_{2} \hat{I}^{2} P q_{1}+a_{1} d_{2} \hat{I}^{2} \hat{P} q_{1}^{2}+a_{1} d_{1} \hat{I}^{2} \hat{P} q_{1} q_{2}+a_{1} \hat{I}^{2} \hat{P} q_{1}^{2} q_{2}+a_{1} d_{1} \hat{I} \hat{P} q_{1} \mu+a_{1} \hat{I} \hat{P} q_{1}^{2} \mu\right)\left(p_{1}-\tau_{1}\right)+$ $\left(\frac{a_{2} d_{2}^{2} \hat{I} \hat{M} \hat{P} r}{K}+\frac{a_{2} d_{2} \hat{I} \hat{M} \hat{P} q_{2} r}{K}+a_{2} d_{1} d_{2} \hat{I} \hat{P} \beta+a_{2} d_{2} \hat{I} \hat{P} q_{1} \beta\right) p_{2}+\left(\frac{a_{2} d_{2} \hat{I} \hat{M} \hat{P} q_{2} r}{K}+\frac{a_{2} \hat{I} \hat{M} \hat{P} q_{2}^{2} r}{K}+a_{2} d_{1} \hat{I} \hat{P} q_{2} \beta+\right.$ $\left.a_{2} \hat{I} \hat{P} q_{1} q_{2} \beta\right)\left(p_{2}-\tau_{2}\right)$.

Now, on applying the Routh-Hurwitz criteria, the system is locally stable as all the coefficients $Z_{1}, Z_{2}$, and $Z_{3}$ are positive and the condition

$$
Z_{1} Z_{2}-Z_{3}>0
$$

is satisfied. Hence, the interior equilibrium is locally asymptotically stable as all the conditions of Routh-Hurwitz criteria are satisfied.

Remark We have considered the tax $\tau_{1}, \tau_{2}$ per unit of forestry biomass is less than the price $p_{1}, p_{2}$ per unit of forestry biomass. Hence, if the co-existence state of the system exists, it is always stable for the system. 


\subsubsection{System with maturation delay}

For the analysis of delayed system, we refer to [7, 16, 17]. Equilibrium points of the system without maturation delay and of that with maturation delay are the same, as time delay does not change the equilibria of the system. To study the local stability of the interior equilibrium $\hat{E}=(\hat{P}, \hat{M}, \hat{I})$ for the delay system, first we use linear transformation in the system equations (1)-(3) as $P=P_{1}+\hat{P}, M=M_{1}+\hat{M}$, and $I=I_{1}+\hat{I}$, where $P_{1} \ll 1, M_{1} \ll 1$, $I_{1} \ll 1$. A variational matrix for the delay system about the interior equilibrium is

$$
J_{2}=\left[\begin{array}{ccc}
-\beta e^{-\lambda h_{1}}-d_{1} \hat{I}-q_{1} \hat{I}-\frac{2 r \hat{P}}{K}+r+\gamma & 0 & -\left(d_{1}+q_{1}\right) \hat{P} \\
\beta e^{-\lambda h_{1}} & -\mu-\left(q_{2}+d_{2}\right) \hat{I} & -\left(d_{2}+q_{2}\right) \hat{M} \\
a_{1} \hat{I}\left(d_{1} p_{1}+q_{1}\left(p_{1}-\tau_{1}\right)\right) & a_{2} \hat{I}\left(d_{2} p_{2}+q_{2}\left(p_{2}-\tau_{2}\right)\right) & 0
\end{array}\right],
$$

the characteristic equation corresponding to the interior equilibrium is

$$
\lambda^{3}+A_{1} \lambda^{2}+A_{2} \lambda+A_{3}+\beta e^{-\lambda h_{1}}\left(B_{1} \lambda^{2}+B_{2} \lambda+B_{3}\right)=0
$$

where $A_{1}=\mu-r-\gamma+d_{1} \hat{I}+d_{2} \hat{I}+q_{1} \hat{I}+\frac{2 r \hat{P}}{K}+q_{2} \hat{I}, A_{2}=-r \mu-\gamma \mu-d_{2} r \hat{I}-q_{2} r \hat{I}-d_{2} \gamma \hat{I}-$ $q_{2} \gamma \hat{I}+d_{1} \mu \hat{I}+q_{1} \mu \hat{I}+d_{1} d_{2} \hat{I}^{2}+d_{2} q_{1} \hat{I}^{2}+d 1 q 2 \hat{I}^{2}+q_{1} q_{2} \hat{I}^{2}+a_{2} d_{2}^{2} p_{2} \hat{I} \hat{M}+2 a_{2} d_{2} p_{2} q_{2} \hat{I} \hat{M}+$ $a_{2} p_{2} q_{2}^{2} \hat{I} \hat{M}-a_{2} d_{2} q_{2} \tau_{2} \hat{I} \hat{M}-a_{2} q_{2}^{2} \tau_{2} \hat{I} \hat{M}+\frac{2 r \mu \hat{P}}{K}+a_{1} d_{1}^{2} p_{1} \hat{I} \hat{P}+2 a_{1} d_{1} p_{1} q_{1} \hat{I} \hat{P}+a_{1} p_{1} q_{1}^{2} \hat{I} \hat{P}+\frac{2 d_{2} r \hat{I} \hat{P}}{K}+$ $\frac{2 q_{2} r \hat{I} \hat{P}}{K}-a_{1} d_{1} q_{1} \tau_{1} \hat{I} \hat{P}-a_{1} q_{1}^{2} \tau_{1} \hat{I} \hat{P}, A_{3}=p_{1}\left(a_{1} d_{1}^{2} \mu \hat{I} \hat{P}+a_{1} d_{1} q_{1} \mu \hat{I} \hat{P}+a_{1} d_{1}^{2} d_{2} \hat{I}^{2} \hat{P}+a_{1} d_{1} d_{2} q_{1} \hat{I}^{2} \hat{P}+\right.$ $\left.a_{1} d_{1}^{2} q_{2} \hat{I}^{2} \hat{P}+a_{1} d_{1} q_{1} q_{2} \hat{I}^{2} \hat{P}\right)+\left(p_{1}-\tau_{1}\right)\left(a_{1} d_{1} q_{1} \mu \hat{I} \hat{I}\right)+a_{1} q_{1}^{2} \mu \hat{I} \hat{P}+a_{1} d_{1} d_{2} q_{1} \hat{I}^{2} \hat{P}+a_{1} d_{2} q_{1}^{2} \hat{I}^{2} \hat{P}+$ $\left.a_{1} d_{1} q_{1} q_{2} \hat{I}^{2} \hat{P}+a_{1} q_{1}^{2} q_{2} \hat{I}^{2} \hat{P}\right)+p_{2}\left(-a_{2} d_{2}^{2} r \hat{I} \hat{M}-a_{2} d_{2} q_{2} r \hat{I} \hat{M}-a_{2} d_{2}^{2} \gamma \hat{I} \hat{M}-a_{2} d_{2} q_{2} \gamma \hat{I} \hat{M}\right.$ $+a_{2} d_{1} d_{2}^{2} \hat{I}^{2} \hat{M}+a_{2} d_{2}^{2} q_{1} \hat{I}^{2} \hat{M}+a_{2} d_{1} d_{2} q_{2} \hat{I}^{2} \hat{M}+a_{2} d_{2} q_{1} q_{2} \hat{I}^{2} \hat{M}+\frac{2 a_{2} d_{2}^{2} r \hat{I} \hat{M} \hat{P}}{K}+\frac{2 a_{2} d_{2} q_{2} r \hat{I} \hat{M} \hat{P}}{K}+\left(p_{2}-\right.$ $\left.\tau_{2}\right)\left(a_{2} d_{2} q_{2} r \hat{I} \hat{M}+a_{2} q_{2}^{2} r \hat{I} \hat{M}+a_{2} d_{2} q_{2} \gamma \hat{I} \hat{M}+a_{2} q_{2}^{2} \gamma \hat{I} \hat{M}-a_{2} d_{1} d_{2} q_{2} \hat{I}^{2} \hat{M}-a_{2} d_{2} q_{1} q_{2} \hat{I}^{2} \hat{M}-\right.$ $\left.a_{2} d_{1} q_{2}^{2} \hat{I}^{2} \hat{M}-a_{2} q_{1} q_{2}^{2} \hat{I}^{2} \hat{M}-\frac{2 a_{2} d_{2} q_{2} r \hat{I} \hat{M} \hat{P}}{K}-\frac{2 a_{2} q_{2}^{2} r \hat{I} \hat{M} \hat{P}}{K}\right), B_{1}=\beta, B_{2}=\beta \mu+d_{2} \beta \hat{I}+q_{1} \beta \hat{I}$, and $B_{3}=\beta d_{2} \hat{I} \hat{P}\left(d_{1}+q_{1}\right) p_{2}+a_{2} q_{2} \hat{I} \hat{P}\left(d_{1}+q_{1}\right) \beta\left(p_{2}-\tau_{2}\right)$.

Previously, it was noticed when there is no delay $\left(h_{1}=0\right)$ the system is locally asymptotically stable. Now, we assume for some $\lambda=i \omega$ with $\omega>0$ be the solution of (9). Then equation (9) can be written as

$$
\begin{aligned}
& A_{3}+i A_{2} \omega-A_{1} \omega^{2}-i \omega^{3}+B_{3} \cos \left(\omega h_{1}\right)+i B_{2} \omega \cos \left(\omega h_{1}\right)-B_{1} \omega^{2} \cos \left(\omega h_{1}\right) \\
& -i B_{3} \sin \left(\omega h_{1}\right)+B_{2} \omega \sin \left(\omega h_{1}\right)+i B_{1} \omega^{2} \sin \left(\omega h_{1}\right)=0 .
\end{aligned}
$$

On separating the real and imaginary parts, we have

$$
\begin{aligned}
& A_{3}-A_{1} \omega^{2}=B_{1} \omega^{2} \cos \left(\omega h_{1}\right)-B_{3} \cos \left(\omega h_{1}\right)-B_{2} \omega \sin \left(\omega h_{1}\right) . \\
& A_{2} \omega-\omega^{3}=B_{3} \sin \left(\omega h_{1}\right)-B_{1} \omega^{2} \sin \left(\omega h_{1}\right)-B_{2} \omega \cos \left(\omega h_{1}\right) .
\end{aligned}
$$

Squaring and adding equations (11) and (12) and substituting $\omega^{2}=\zeta$ leads to a cubic equation

$$
h(\zeta)=\zeta^{3}+k_{1} \zeta^{2}+k_{2} \zeta+k_{3}=0
$$

where $k_{1}=A_{2}^{2}-B_{2}^{2}-2 B_{1} B_{3}, k_{2}=A_{1}^{2}-2 A_{2}-B_{1}^{2}$, and $k_{3}=A_{3}^{2}-B_{3}^{2}$. If the condition of the Routh-Hurwitz criterion satisfies $h(\zeta)$, then equation (13) will not have any positive real 
root, and we may not get any positive value of $\omega$, which satisfies equations (11) and (12). In this case the results are given in the form of the following lemma.

Lemma 2 If coefficients of equation (13) are positive, all the conditions of Routh-Hurwitz criteria are satisfied and the interior equilibrium exists, it is locally asymptotically stable for all $h_{1}>0$.

Proof Now, we consider that the values' of $k_{1}, k_{2}$, and $k_{3}$ do not satisfy the conditions of the Routh-Hurwitz criterion. In this case a simple assumption

$$
k_{1}>0, \quad k_{2}>0, \quad \text { and } \quad k_{3}<0
$$

gives that equation (14) has one positive root. If condition (14) holds good, then equation (9) has a pair of purely imaginary roots $\pm i \omega_{0}$. Next, using transcendental equations (11)(12), we get

$$
\tan \omega h_{1}=\frac{B_{2} \omega_{0}\left(A_{3}-A_{1} \omega_{0}^{2}\right)-\left(A_{2} \omega_{0}-\omega_{0}^{3}\right)\left(B_{3}-B_{1} \omega_{0}^{2}\right)}{B_{2} \omega_{0}\left(A_{2} \omega_{0}-\omega_{0}^{3}\right)+\left(A_{3}-A_{1} \omega_{0}^{2}\right)\left(B_{3}-B_{1} \omega_{0}^{2}\right)}
$$

For a positive value of $\omega_{0}$, the value of $h_{1}$ is defined as

$$
h_{1 n}=\frac{n \pi}{\omega_{0}}+\frac{1}{\omega_{0}} \tan ^{-1} \frac{B_{2} \omega_{0}\left(A_{3}-A_{1} \omega_{0}^{2}\right)-\left(A_{2} \omega_{0}-\omega_{0}^{3}\right)\left(B_{3}-B_{1} \omega_{0}^{2}\right)}{B_{2} \omega_{0}\left(A_{2} \omega_{0}-\omega_{0}^{3}\right)+\left(A_{3}-A_{1} \omega_{0}^{2}\right)\left(B_{3}-B_{1} \omega_{0}^{2}\right)}
$$

for $n=0,1,2, \ldots$.

In the similar logic $[17,18]$, it can be concluded that the stable interior equilibrium $\hat{E}$ remains stable for $h_{1}<h_{10}$. Next, we will investigate the possibility of Hopf bifurcation as $h_{1}$ is greater than $h_{10}$.

Lemma 3 The transversality condition $\frac{d \operatorname{Re} \lambda_{k}\left(h_{1 k}\right)}{d \tau}>0$, where $k=0,1,2,3, \ldots$, is satisfied, i.e., system (1)-(3) undergoes a Hopf bifurcation at the co-existing state of equilibrium $\hat{E}$ for $h_{1}=h_{1 n}$.

Proof Differentiating (9) with respect to $h_{1}$ gives

$$
\left(\frac{d \lambda}{d h_{1}}\right)^{-1}=\frac{\left(3 \lambda^{2}+2 A_{1} \lambda+A_{2}\right)+\left(2 B_{1} \lambda+B_{2}\right) e^{-\lambda h_{1}}}{\lambda\left(B_{1} \lambda^{2}+B_{2} \lambda+B_{3}\right) e^{\lambda h_{1}}}-\frac{h_{1}}{\lambda} .
$$

Now,

$$
\begin{aligned}
\operatorname{sgn}\left[\frac{d(\operatorname{Re}(\lambda))}{d h_{1}}\right]_{h_{1}=h_{10}} & =\operatorname{sgn}\left[\frac{d(\operatorname{Re}(\lambda))}{d h_{1}}\right]_{h_{1}=h_{10}}^{-1} \\
& =\operatorname{sgn}\left[\operatorname{Re}\left(\frac{d(\lambda)}{d h_{1}}\right)^{-1}\right]_{\lambda=i \omega_{0}} \\
& =\operatorname{sgn}\left[\frac{3 \omega_{0}^{4}+2 k_{1} \omega_{0}^{2}+k_{2}}{B_{2}^{2} \omega_{0}^{2}+\left(B_{3}-B_{1} \omega_{0}^{2}\right)^{2}}\right] \\
& =\operatorname{sgn}\left[\frac{h^{\prime}\left(\omega_{0}^{2}\right)}{B_{2}^{2} \omega_{0}^{2}+\left(B_{3}-B_{1} \omega_{0}^{2}\right)^{2}}\right] .
\end{aligned}
$$


The condition defined in equation (15) is satisfied, $h^{\prime}\left(\omega_{0}^{2}\right)>0$. Hence the transversality condition holds, which confirms that the Hopf bifurcation occurs at $h_{1}=h_{10}$.

Remark The condition defined in equation (8) is satisfied when equation (15) holds, the interior equilibrium $\hat{E}$ of system (1)-(3) is locally asymptotically stable for $h_{1} \epsilon\left[0, h_{10}\right)$ and becomes unstable for $h_{1}>h_{10}$.

\subsubsection{Stability and direction of a Hopf bifurcation point}

Previously, we have acquired the condition where the system undergoes bifurcation for the interior equilibrium when $h_{1}$ crosses the critical threshold. Here, we will derive explicit formulae for determining the direction and bifurcating periodic solutions that emerge through the Hopf bifurcation. We will use the normal form theory and the center manifold theorem as in [19]. Without loss of generality, we denote the critical values of $h$ by $h_{k}$ at which (9) has a pair of purely imaginary roots and the system undergoes the Hopf bifurcation. Hence, for any root of (9) of the form $\psi(h)=v(h)+i \omega(h), v\left(h_{k}\right)=0, \omega\left(h_{k}\right)=\omega_{0}$, and $\left|\frac{d v}{d h}\right|_{h=h_{k}} \neq 0$, let $h=h_{k}+\mu, \mu \in R$, so that $\mu=0$ is a Hopf bifurcation value for the system.

Next, taking the space of continuous real-valued functions as $C=C\left([-1,0], R^{3}\right)$, using the transformation $u_{1}=P(t)-\hat{P}, u_{2}=M(t)-\hat{M}, u_{3}=I(t)-\hat{I}$, and $\chi_{i}(t)=u_{i}(h t)$ for $i=1,2,3$, the delay system (1)-(3) then transforms to FDE in $C$ as follows:

$$
\frac{d \chi}{d t}=L_{\mu} \chi_{t}+f\left(\mu, \chi_{t}\right)
$$

where $\chi(t)=\left(\chi_{1}(t), \chi_{2}(t), \chi_{3}(t)\right)^{T} \in R^{3}, \chi_{t}(\Theta)=\chi(t+\Theta), \Theta \in[-1,0], L_{\mu}: C \rightarrow R^{3}$, and $f$ : $C \times R \rightarrow R^{3}$ are given by

$$
L_{\mu} \zeta=\left(h_{k}+\mu\right)\left[M_{1} \zeta(0)+M_{2} \zeta(-1)\right]
$$

where

$$
\begin{aligned}
& M_{1}=\left(\begin{array}{ccc}
r+\gamma-\frac{2 r \hat{P}}{K}-q_{1} \hat{I}-d_{1} \hat{I} & 0 & -\left(q_{1}+d_{1}\right) \hat{P} \\
0 & -\left(q_{2} \hat{I}+d_{2} \hat{I}+\mu\right\} & -\left(q_{2}+d_{2}\right) \hat{M} \\
\left\{a_{1} q_{1}\left(p_{1}-\tau_{1}\right)+a_{1} p_{1} d_{1}\right\} \hat{I} & \left\{a_{2} q_{2}\left(p_{2}-\tau_{2}\right)+a_{2} p_{2} d_{2}\right\} \hat{I} & 0
\end{array}\right), \\
& M_{2}=\left(\begin{array}{ccc}
-\beta & 0 & 0 \\
\beta & 0 & 0 \\
0 & 0 & 0
\end{array}\right)
\end{aligned}
$$

and

$$
f(\mu, \zeta)=\left(h_{k}+\mu\right)\left(\begin{array}{c}
Z_{1} \\
Z_{2} \\
Z_{3}
\end{array}\right)
$$

where $Z_{1}=-\frac{r}{K} \zeta_{1}^{2}(0)-\left(q_{1}+d_{1}\right) \zeta_{1}(0) \zeta_{3}(0), Z_{2}=-\left(q_{2}+d_{2}\right) \zeta_{2}(0) \zeta_{3}(0), Z_{3}=\left[a_{1}\left\{\left(p_{1}-\tau_{1}\right) q_{1}+\right.\right.$ $\left.\left.p_{1} d_{1}\right\}\right] \zeta_{1}(0) \zeta_{3}(0)+\left[a_{2}\left\{\left(p_{2}-\tau_{2}\right) q_{2}+p_{2} d_{2}\right\}\right] \zeta_{2}(0) \zeta_{3}(0)$ for $\zeta=\left(\zeta_{1} \zeta_{2}, \zeta_{3}\right)^{T} \in C$. 
As per the Riesz representation theorem, there exists a function $\eta(\Theta, \mu)$ whose components are of bounded variation for $\Theta \in[-1,0]$ such that

$$
L_{\mu} \zeta=\int_{0}^{-1} d \eta(\Theta, \mu) \zeta(\Theta)
$$

In view of equation (17), we can choose

$$
\eta(\Theta, \mu)=\left(h_{k}+\mu\right)\left[M_{1} \delta(\Theta)+M_{2} \delta(\theta+1)\right]
$$

where $\zeta \in C^{1}\left([-1,0], R^{3}\right)$, define

$$
A(\mu) \zeta= \begin{cases}\frac{d \zeta(\Theta)}{d \Theta}, & \Theta \in[-1,0) \\ \int_{-1}^{0} d \eta(\rho, \mu) \zeta(\rho)=L_{\mu} \zeta, & \Theta=0\end{cases}
$$

Also,

$$
R(\mu) \zeta= \begin{cases}0, & \Theta \in[-1,0) \\ f(\zeta, \mu), & \Theta=0\end{cases}
$$

System (16) is equivalent to

$$
\dot{\chi}_{t}=A(\mu) \chi_{t}+R(\mu) \chi_{t}
$$

where $\chi_{t}(\Theta)=\chi(t+\Theta)$ for $\Theta \in[-1,0]$.

For $\psi \in C^{1}\left([-1,0],\left(R^{3}\right)^{*}\right)$, define

$$
A^{*} \xi(\rho)= \begin{cases}-\frac{d \xi(\rho)}{d s}, & \rho \in(0,1] \\ \int_{-1}^{0} d \eta^{T}(t, 0) \xi(-t), & \rho=0\end{cases}
$$

and a bilinear inner product

$$
\langle\xi, \zeta\rangle=\bar{\xi}(0) . \zeta(0)-\int_{\Theta=-1}^{0} \int_{\nu=0}^{\Theta} \bar{\xi}^{T}(\nu-\Theta) d \eta(\Theta) \zeta(\nu) d \nu .
$$

Here, $\eta(\Theta)=\eta(\Theta, 0)$, then $A(0)$ (from here onwards we use $A(0)$ as $A$ ) and $A^{*}$ are adjoint operators. Since $\pm i \omega_{0} h_{k}$ are the eigenvalues of $A$, they are also the eigenvalues of $A^{*}$. Next, we compute the eigenvectors of $A$ and $A^{*}$ corresponding to $+i \omega_{0} h_{k}$ and $-i \omega_{0} h_{k}$, respectively.

Suppose $q(\Theta)=\left(1, b_{1}, b_{2}\right)^{T} e^{i \omega_{0} h_{k} \Theta}$ is the eigenvector of $A$ corresponding to eigenvalues $i \omega_{0} h_{k}$, then

$$
A q(\Theta)=i \omega_{0} h_{k} q(\Theta)
$$

which for $\Theta=0$ gives

$$
h_{k}\left(\begin{array}{ccc}
m_{11} & 0 & m_{13} \\
m_{21} & m_{22} & m_{23} \\
m_{31} & m_{32} & m_{33}
\end{array}\right) q(0)=\left(\begin{array}{l}
0 \\
0 \\
0
\end{array}\right) \text {, }
$$


where $m_{11}=i \omega_{0}+\beta e^{-i \omega_{0} h_{k}}-(r+\gamma)+\left(\frac{2 r \hat{P}}{K}+q_{1} \hat{I}+d_{1} \hat{I}\right), m_{13}=\left(q_{1}+d_{1}\right) \hat{P}, m_{21}=-\beta e^{-i \omega_{0} h_{k}}$, $m_{22}=i \omega_{0}+q_{2} \hat{I}+d_{2} \hat{I}+\mu, m_{23}=\left(q_{2}+d_{2}\right) \hat{M}, m_{31}=-\left[a_{1}\left\{\left(p_{1}-\tau_{1}\right) q_{1}+p_{1} d_{1}\right\}\right] \hat{I}, m_{32}=$ $-\left[a_{2}\left\{\left(p_{2}-\tau_{2}\right) q_{2}+p_{2} d_{2}\right\}\right] \hat{I}, m_{33}=i \omega_{0}$.

Solving the system of equation (29), we get $b_{1}=\frac{i \omega_{0} a_{2}-\left[a_{1}\left\{\left(p_{1}-\tau_{1}\right) q_{1}+p_{1} d_{1}\right\}\right] \hat{I}}{\left[a_{2}\left\{\left(p_{2}-\tau_{2}\right) q_{2}+p_{2} d_{2}\right\}\right] \hat{I}}, \quad b_{2}=$ $-\frac{i \omega_{0}+\beta e^{-i \omega_{0} h_{k}+\left(\frac{2 r \hat{P}}{K}+q_{1} \hat{I}+d_{1} \hat{I}\right)-r-\gamma}}{\left(q_{1}+d_{1}\right) \hat{P}}$.

Similarly, we calculate $q^{*}(\rho)=\left(1, b_{1}^{*}, b_{2}^{*}\right)^{T} e^{i \omega_{0} h_{k} \rho}$ such that

$$
A^{*} q^{*}(\rho)=-i \omega_{0} h_{k} q^{*}(\rho)
$$

where $b_{1}^{*}=\frac{C_{2} b_{2}^{*}}{C_{1}}, b_{2}^{*}=\frac{C_{1}\left(q_{1}+d_{1}\right) \hat{P}}{C_{1} \omega_{0}-C_{2}\left(q_{2}+d_{2}\right) \hat{M}}, C_{1}=-i \omega_{0}+\left\{\left(q_{2}+d_{2}\right) \hat{I}+\mu\right\}, C_{2}=\left[a_{2}\left\{\left(p_{2}-\tau_{2}\right) q_{2}+\right.\right.$ $\left.\left.p_{2} d_{2}\right\}\right] \hat{I}$.

The normalization condition gives $\left\langle q^{*}(\rho) \cdot q(\Theta)\right\rangle=1, \bar{q}^{*}(0) \cdot q(0)-\bar{D} \int_{\Theta=-1}^{0} \int_{v=0}^{\Theta} \bar{q}^{*}(0) \times$ $e^{-i \omega_{0} h_{k}(v-\Theta)} d \eta(\Theta) q(0) e^{i \omega_{0} h_{k} v} d v=1, \bar{D}\left[1+b_{1} \bar{b}_{1}^{*}+b_{2} \bar{b}_{2}^{*}-e^{i \omega_{0} h_{k}} h_{k} \phi \bar{b}_{2}^{*}\right]=1$.

Thus, $\bar{D}$ is chosen such that

$$
\bar{D}=\frac{1}{1+b_{1} \bar{b}_{1}^{*}+b_{2} \bar{b}_{2}^{*}-e^{i \omega_{0} h_{k} h_{k} \phi \bar{b}_{2}^{*}} .}
$$

Proceeding the same as in [19] and using the same notation, we compute the coordinates to describe the center manifold $C_{0}$ at $\mu=0$. Let $\chi_{t}$ be a solution of equation (25) when $\mu=0$. Define

$$
Z(t)=\left\langle q^{*}, \chi_{t}\right\rangle, \quad W(t, \Theta)=\chi_{t}(\Theta)-2 \operatorname{Re}\{Z(t) q(\Theta)\}
$$

On the center manifold $C_{0}$, we have

$$
\begin{aligned}
& W(t, \theta)=W(Z, \bar{Z}, \Theta) \\
& W(z, \bar{z}, \Theta)=W_{20}(\Theta) \frac{z^{2}}{2}+W_{11}(\Theta) z \bar{z}+W_{02}(\Theta) \frac{\bar{z}^{2}}{2}+\cdots
\end{aligned}
$$

$z$ and $\bar{z}$ are local coordinates for the center manifold $C_{0}$ in the direction of $q^{*}$ and $\bar{q}^{*}$. Note that $W$ is real if $\chi_{t}$ is real. Here, we consider only real solutions. For solution $\chi_{t} \in C_{0}$ of equation (25), since $\mu=0$, we have

$$
\begin{aligned}
\dot{z} & =i \omega_{0} h_{k} z+\bar{q}^{*}(0) \cdot f(0, W(z, \bar{z}, 0)+2 \operatorname{Re}\{z q(0)\}) \\
& =i \omega_{0} h_{k} z+\bar{q}^{*}(0) \cdot f_{0}(z, \bar{z}) .
\end{aligned}
$$

We can rewrite this equation as

$$
\begin{aligned}
& \dot{z}=i \omega_{0} h_{k} z+g(z, \bar{z}), \quad \text { where } g(z, \bar{z})=\bar{q}^{*}(0) \cdot f_{0}(z, \bar{z}) \\
& \text { or }=g_{20} \frac{z^{2}}{2}+g_{11} z \bar{z}+g_{02} \frac{\bar{z}^{2}}{2}+g_{21} \frac{z^{2} \bar{z}}{2}+\cdots
\end{aligned}
$$

It follows from (33) and (35) that

$$
\chi_{t}(\Theta)=W(z, \bar{z}, \Theta)+2 \operatorname{Re}\{z q(\Theta)\}
$$




$$
\begin{aligned}
\text { or }= & W_{20}(\Theta) \frac{z^{2}}{2}+W_{11}(\Theta) z \bar{z}+W_{02}(\Theta) \frac{\bar{z}^{2}}{2}+z\left(1, b_{1}, b_{2}\right)^{T} \\
& \times e^{i \omega_{0} h_{k} \Theta}+\bar{z}\left(1, \overline{b_{1}}, \overline{b_{2}}\right)^{T} e^{-i \omega_{0} h_{k} \Theta}+\cdots
\end{aligned}
$$

Also, we have

$$
g(z, \bar{z})=\bar{q}^{*}(0) \cdot f_{0}\left(0, \chi_{t}\right)=h_{k} \bar{D}\left(1,{\overline{b_{1}}}^{*},{\overline{b_{2}}}^{*}\right)^{T}\left(\begin{array}{c}
V_{1} \\
V_{2} \\
V_{3}
\end{array}\right)
$$

where $V_{1}=-\frac{r}{K} \chi_{1 t}^{2}(0)-\left(q_{1}+d_{1}\right) \chi_{1 t}(0) \chi_{3 t}(0), V_{2}=-\left(q_{2}+d_{2}\right) \chi_{2 t}(0) \chi_{3 t}(0), V_{3}=\left[a_{1}\left\{\left(p_{1}-\right.\right.\right.$ $\left.\left.\left.\tau_{1}\right) q_{1}+p_{1} d_{1}\right\}\right] \chi_{1 t}(0) \chi_{3 t}(0)+\left[a_{2}\left\{\left(p_{2}-\tau_{2}\right) q_{2}+p_{2} d_{2}\right\}\right] \chi_{2 t}(0) \chi_{3 t}(0)$, so that

$$
\begin{aligned}
& \chi_{1 t}(\Theta)=W_{20}^{1}(\Theta) \frac{z^{2}}{2}+W_{11}^{1}(\Theta) z \bar{z}+W_{02}^{1}(\Theta) \frac{\bar{z}^{2}}{2}+z e^{i \omega_{0} h_{k} \Theta}+\bar{z} e^{-i \omega_{0} h_{k} \Theta}+\cdots \\
& \chi_{2 t}(\Theta)=W_{20}^{2}(\Theta) \frac{z^{2}}{2}+W_{11}^{2}(\Theta) z \bar{z}+W_{02}^{2}(\Theta) \frac{\bar{z}^{2}}{2}+b_{1} z e^{i \omega_{0} h_{k} \Theta}+\overline{b_{1}} \bar{z} e^{-i \omega_{0} h_{k} \Theta}+\cdots \\
& \chi_{3 t}(\Theta)=W_{20}^{3}(\Theta) \frac{z^{2}}{2}+W_{11}^{3}(\Theta) z \bar{z}+W_{02}^{3}(\Theta) \frac{\bar{z}^{2}}{2}+b_{2} z e^{i \omega_{0} h_{k} \Theta}+\bar{z} \overline{b_{2}} e^{-i \omega_{0} h_{k} \Theta}+\cdots
\end{aligned}
$$

Therefore,

$$
\begin{aligned}
\chi_{1 t}(0)= & z+\bar{z}+W_{20}^{1}(0) \frac{z^{2}}{2}+W_{11}^{1}(0) z \bar{z}+W_{02}^{1}(0) \frac{\bar{z}^{2}}{2}+\cdots \\
\chi_{2 t}(0)= & b_{1} z+\overline{b_{1}} \bar{z}+W_{20}^{2}(0) \frac{z^{2}}{2}+W_{11}^{2}(0) z \bar{z}+W_{02}^{1}(0) \frac{\bar{z}^{2}}{2}+\cdots, \\
\chi_{3 t}(0)= & b_{2} z+\overline{b_{2}} \bar{z}+W_{20}^{3}(0) \frac{z^{2}}{2}+W_{11}^{3}(0) z \bar{z}+W_{02}^{3}(0) \frac{\bar{z}^{2}}{2}+\cdots, \\
g(z, \bar{z})= & h_{k} \bar{D}\left(1,{\overline{b_{1}}}^{*},{\overline{b_{2}}}^{*}\right)^{T} \cdot\left(\begin{array}{c}
V_{1} \\
V_{2} \\
V_{3}
\end{array}\right) \\
= & h_{k} \bar{D}\left[-\frac{r}{K} \chi_{1 t}^{2}(0)+\left\{-\left(q_{1}+d_{1}\right)+a_{1}{\overline{b_{2}}}^{*}\left(\left(p_{1}-\tau_{1}\right) q_{1}+p_{1} d_{1}\right)\right\} \chi_{1 t}(0) \chi_{3 t}(0)\right] \\
& +h_{k} \bar{D}\left[\left\{-\bar{b}_{1}^{*}\left(q_{2}+d_{2}\right)+a_{2} \bar{b}_{2}{ }^{*}\left\{\left(p_{2}-\tau_{2}\right) q_{2}+p_{2} d_{2}\right\}\right\} \chi_{2 t}(0) \chi_{3 t}(0)\right] .
\end{aligned}
$$

Comparing the coefficients in (37) with those in (41), we get

$$
\begin{aligned}
g_{20}= & 2 h_{k} \bar{D}\left[-\frac{r}{K}-b_{2}\left(q_{1}+d_{1}\right)+b_{2} \bar{b}_{2}^{*}+a_{1}\left\{\left(p_{1}-\tau_{1}\right) q_{1}+p_{1} d_{1}\right\}\right. \\
& \left.-b_{1} b_{2}{\overline{b_{1}}}^{*}\left(q_{2}+d_{2}\right)+b_{1} b_{2} \bar{b}_{2}^{*} a_{2}\left\{\left(p_{2}-\tau_{2}\right) q_{2}+p_{2} d_{2}\right\}\right] \\
g_{11}= & 2 h_{k} \bar{D}\left[-\frac{r}{K}+\operatorname{Re}\left(b_{2}\right)\left\{-\left(q_{1}+d_{1}\right)+\bar{b}_{2}^{*} a_{1}\left(\left(p_{1}-\tau_{1}\right) q_{1}+p_{1} d_{1}\right)\right\}\right] \\
& +2 h_{k} \bar{D}\left[\left(\overline{b_{1}} b_{2}+b_{1} \bar{b}_{2}\right)\left\{-\bar{b}_{1}^{*}\left(q_{2}+d_{2}\right)+\bar{b}_{2}^{*} a_{2}\left(\left(p_{2}-\tau_{2}\right) q_{2}+p_{2} d_{2}\right)\right\}\right], \\
g_{02}= & 2 h_{k} \bar{D}\left[-\frac{r}{K}-\bar{b}_{2}\left(q_{1}+d_{1}\right)+\bar{b}_{2} \bar{b}_{2}^{*} a_{1}\left\{\left(p_{1}-\tau_{1}\right) q_{1}+p_{1} d_{1}\right\}\right]
\end{aligned}
$$




$$
\begin{aligned}
& +2 h_{k} \bar{D}\left[\bar{b}_{1} \bar{b}_{2}\left\{-\bar{b}_{1}^{*}\left(q_{2}+d_{2}\right)+a_{2} \bar{b}_{2}^{*}\left(\left(p_{2}-\tau_{2}\right) q_{2}+p_{2} d_{2}\right)\right\}\right] . \\
g_{21}= & 2 h_{k} \bar{D}\left[-\frac{r}{K}\left(W_{20}^{(1)}+2 W_{11}^{(1)}\right)+\left\{-\left(q_{1}+d_{1}\right)+a_{1}{\overline{b_{2}}}^{*}\left(\left(p_{1}-\tau_{1}\right) q_{1}\right.\right.\right. \\
& \left.\left.\left.+p_{1} d_{1}\right)\right\}\left(b_{2} W_{11}^{(1)}+\frac{\bar{b}_{2}}{2} W_{20}^{(1)}+\frac{W_{20}^{(3)}}{2}+W_{11}^{(3)}\right)\right]+2 h_{k} \bar{D}\left[-{\overline{b_{1}}}^{*}\left(q_{2}+d_{2}\right)\right. \\
& \left.+a_{2} \bar{b}_{2}^{*}\left\{\left(p_{2}-\tau_{2}\right) q_{2}+p_{2} d_{2}\right\}\left(b_{2} W_{11}^{(2)}+\bar{b}_{2} W_{20}^{(2)}+\frac{\bar{b}_{1} W_{20}^{(3)}}{2}+b_{1} W_{11}^{(3)}\right)\right] .
\end{aligned}
$$

In order to compute (43), we need $W_{20}(\Theta)$ and $W_{11}(\Theta)$. From equations (33) and (37), we have

$$
\begin{aligned}
\dot{W} & =\dot{\chi}_{t}-\dot{z} q-\dot{z} \bar{q} \\
& = \begin{cases}A W-2 \operatorname{Re}\left\{\bar{q}^{*}(0)(0) \cdot f_{0} q(\Theta)\right\}, & \Theta \in[-1,0), \\
A W-2 \operatorname{Re}\left\{\bar{q}^{*}(0)(0) \cdot f_{0} q(0)\right\}+f_{0}, & \Theta=0\end{cases} \\
& =A W+H(z, \bar{z}, \Theta)
\end{aligned}
$$

with

$$
H(z, \bar{z}, \Theta)=H_{20}(\Theta) \frac{z^{2}}{2}+H_{11}(\Theta) z \bar{z}+H_{02}(\Theta) \frac{\bar{z}^{2}}{2}+\cdots .
$$

Also, on $C_{0}$, using the chain rule, we get

$$
\dot{W}=W_{z} \dot{z}+W_{\bar{z}} \dot{\bar{z}}
$$

It follows from (54), (44), and (46) that

$$
\begin{aligned}
& \left(A-2 i \omega_{0} h_{k}\right) W_{20}=-H_{20}, \\
& A W_{11}=H_{11} .
\end{aligned}
$$

Now, for $\Theta \in[-1,0)$, we have

$$
\begin{aligned}
H(z, \bar{z}, \Theta) & =-\bar{q}^{*}(0) \cdot f_{0} q(\Theta)-\bar{q}^{*}(0) \cdot \bar{f}_{0} \bar{q}(\Theta) \\
& =-g(z, \bar{z}) q(\Theta)-\bar{g}(z, \bar{z}) \bar{q}(\Theta) \\
& =-\left(g_{20} q(\Theta)+\overline{g_{02}} \bar{q}(\Theta)\right) \frac{z^{2}}{2}-\left(g_{11} q(\Theta)+\bar{g}_{11} \bar{q}(\Theta)\right) z \bar{z}+\cdots,
\end{aligned}
$$

which on computing the coefficients with (45) gives

$$
\begin{aligned}
& H_{20}(\Theta)=-g_{20} q(\Theta)-\overline{g_{02}} \bar{q}(\Theta), \\
& H_{11}(\Theta)=-g_{11} q(\Theta)-\overline{g_{11}^{-}} \bar{q}(\Theta) .
\end{aligned}
$$

From (48), (51), and the definition of $A$, we have

$$
W_{20}^{\prime}(\Theta)=2 i \omega_{0} h_{k} W_{20}(\Theta)+g_{20} q(\Theta)+\overline{g_{02}} \bar{q}(\Theta) .
$$


Note that $q(\Theta)=q(0) e^{i \omega_{0} h_{k} \Theta}$, hence

$$
W_{20}(\Theta)=\frac{i g_{20}}{\omega_{0} h_{k}} q(\Theta)+\frac{i g_{20}^{-}}{3 \omega_{0} h_{k}} \bar{q}(\Theta)+F_{1} e^{2 i \omega_{0} h_{k} \Theta} .
$$

Similarly, from (48), (51), and the definition of $A$, we get

$$
\begin{aligned}
& W_{11}^{\prime}(\Theta)=g_{11} q(\Theta)+\overline{g_{11}} \bar{q}(\Theta), \\
& W_{11}(\Theta)=-\frac{i g_{11}}{\omega_{0} h_{k}} q(\Theta)+\frac{i g_{11}^{-}}{\omega_{0} h_{k}} \bar{q}(\Theta)+F_{2},
\end{aligned}
$$

where $F_{1}=\left(F_{1}^{(1)}, F_{1}^{(2)}, F_{1}^{(3)}\right)$ and $F_{2}=\left(F_{2}^{(1)}, F_{2}^{(2)}, F_{2}^{(3)}\right) \in R^{3}$ are constant vectors to be determined. It follows from the definition of $A$ and (52) that

$$
\begin{aligned}
& \int_{-1}^{0} d \eta(\Theta) W_{20}(\Theta)=2 i \omega_{0} h_{k} W_{20}(0)-H_{20}(0), \\
& \int_{-1}^{0} d \eta(\Theta) W_{11}(\Theta)=-H_{11}(0) .
\end{aligned}
$$

From equations (53) and (55), we get

$$
\begin{aligned}
H_{20}(0)= & -g_{20} q(0)-\overline{g_{02}^{-} q \overline{(0)}} \\
& +2 h_{k}\left(\begin{array}{c}
-\frac{r}{K}+b_{2}\left(q_{1}+d_{1}\right) \\
-b_{1} b_{2}\left(q_{2}+d_{2}\right) \\
b_{2} a_{1}\left(p_{1}-\tau_{1}\right) q_{1}+p_{1} d_{1}+b_{1} b_{2} a_{2}\left(p_{2}-\tau_{2}\right) q_{2}+p_{2} d_{2}
\end{array}\right)
\end{aligned}
$$

and

$$
\begin{aligned}
H_{11}(0)= & -g_{11} q(0)-\overline{g_{11}^{-} q \overline{(0)}} \\
& +2 h_{k}\left(\begin{array}{c}
-\frac{r}{K}+\operatorname{Re}\left(b_{2}\right)\left(q_{1}+d_{1}\right) \\
-\left(\overline{b_{1}} b_{2}+\bar{b}_{1} \bar{b}_{2}\right)\left(q_{2}+d_{2}\right) \\
\operatorname{Re}\left(b_{2}\right) a_{1}\left(p_{1}-\tau_{1}\right) q_{1}+p_{1} d_{1}+\left(\overline{b_{1}} b_{2}+b_{1} \bar{b}_{2}\right) a_{2}\left\{\left(p_{2}-\tau_{2}\right) q_{2}+p_{2} d_{2}\right\}
\end{array}\right) .
\end{aligned}
$$

Using (53) and (58) in (56) and noting that $q(\Theta)$ is an eigenvector of $A$, we have

$$
\begin{aligned}
& \left(2 i \omega_{0} h_{k} I-\int_{1}^{0} e^{2 i \omega_{0} h_{k} \Theta} d \eta(\Theta)\right) F_{1} \\
& =2 h_{k}\left(\begin{array}{cc}
-\left\{\frac{r}{K}+b_{2}\left(q_{1}+d_{1}\right)\right\} \\
-b_{1} b_{2}\left(q_{2}+d_{2}\right) \\
b_{2} a_{1}\left\{\left(p_{1}-\tau_{1}\right) q_{1}+p_{1} d_{1}\right\}+b_{1} b_{2} a_{2}\left\{\left(p_{2}-\tau_{2}\right) q_{2}+p_{2} d_{2}\right\}
\end{array}\right) \\
& \left(\begin{array}{ccc}
2 i \omega_{0}+\beta e^{-i \omega_{0} h_{k}-(r+\gamma)+\frac{2 r \hat{P}}{K}+q_{1} \hat{I}+d_{1} \hat{I}} & 0 \\
-\beta e^{-i \omega_{0} h_{k}} & 2 i \omega_{0}+q_{2} \hat{I}+d_{2} \hat{I}+\mu & \left(q_{1}+d_{1}\right) \hat{I} \\
-\left\{a_{1} q_{1}\left(p_{1}-\tau_{1}\right)+a_{1} p_{1} d_{1}\right\} \hat{I} & -\left\{a_{2} q_{2}\left(p_{2}-\tau_{2}\right)+a_{2} p_{2} d_{2}\right\} \hat{I} & 2 i \omega_{0}
\end{array}\right) \\
& \times\left(\begin{array}{ccc}
F_{1}^{(1)} \\
F_{1}^{(2)} \\
F_{1}^{(3)}
\end{array}\right)=2\left(\begin{array}{cc}
K \\
b_{2} a_{1}\left\{\left(p_{1}-\tau_{1}\right) q_{1}+p_{1} d_{1}\right\}+b_{1} b_{2} a_{2}\left\{\left(p_{2}-\tau_{2}\right) q_{2}+p_{2} d_{2}\right\}
\end{array}\right)
\end{aligned}
$$


Similarly, using (55) and (56) in (59), we get

$$
\begin{aligned}
& \left(\begin{array}{ccc}
\beta-(r+\gamma)+\frac{2 r \hat{P}}{K}+q_{1} \hat{I}+d_{1} \hat{I} & 0 & \left(q_{1}+d_{1}\right) \hat{P} \\
-\beta & q_{2} \hat{I}+d_{2} \hat{I}+\mu & \left(q_{2}+d_{2}\right) \hat{M} \\
-\left\{a_{1} q_{1}\left(p_{1}-\tau_{1}\right)+a_{1} p_{1} d_{1}\right\} \hat{I} & -\left\{a_{2} q_{2}\left(p_{2}-\tau_{2}\right)+a_{2} p_{2} d_{2}\right\} & 0
\end{array}\right)\left(\begin{array}{c}
F_{2}^{(1)} \\
F_{2}^{(2)} \\
F_{2}^{(3)}
\end{array}\right) \\
& =2\left(\begin{array}{c}
-\left\{\frac{r}{K}+\operatorname{Re}\left(b_{2}\right)\left(q_{1}+d_{1}\right)\right\} \\
-\left(\overline{b_{1}} b_{2}+b_{1} \bar{b}_{2}\right)\left(q_{2}+d_{2}\right) \\
\operatorname{Re}\left(b_{2}\right) a_{1}\left\{\left(p_{1}-\tau_{1}\right) q_{1}+p_{1} d_{1}\right\}+\left(\overline{b_{1}} b_{2}+b_{1} \overline{b_{2}}\right) a_{2}\left\{\left(p_{2}-\tau_{2}\right) q_{2}+p_{2} d_{2}\right\}
\end{array}\right) .
\end{aligned}
$$

Next, solving (60) for $F_{1}$ and (61) for $F_{2}$ and using these values, we can determine $W_{20}$ and $W_{11}$, and hence $g_{21}$. Afterwards, to determine the direction, stability, and the period of bifurcating solutions from a critical point at the critical threshold $h=h_{k}$, we can come to the following necessary quantities as given by [19]:

$$
\begin{aligned}
& b_{1}(0)=\frac{i}{2 \omega_{0} h_{k}}\left(g_{11} g_{20}-2\left|g_{11}\right|^{2}-\frac{\left|g_{02}\right|^{2}}{3}\right)+\frac{g_{21}}{2}, \\
& \mu_{2}=-\frac{\operatorname{Re}\left\{b_{1}(0)\right\}}{\operatorname{Re}\left\{\psi^{\prime}\left(h_{k}\right)\right\}} \\
& m_{2}=2 \operatorname{Re}\left\{b_{1}(0)\right\}, \\
& T_{2}=-\frac{I_{m} b_{1}(0)+\mu I_{m}\left\{\psi^{\prime}\left(h_{k}\right)\right\}}{\omega_{0} h_{k}} .
\end{aligned}
$$

Hence, using the results of [19], we have the following theorem.

Theorem 1 If $\mu_{2}>0\left(\mu_{2}<0\right)$, then the Hopf bifurcation is supercritical (subcritical), and the bifurcating periodic solutions exist for $h>h_{k}\left(h<h_{k}\right)$. The bifurcating periodic solution is stable (unstable) if $m_{2}<0\left(m_{2}>0\right)$, and the period increases (decreases) if $T_{2}>0\left(T_{2}<0\right)$.

\section{Bionomic equilibria}

The total net economic revenue at any time is given by $\Pi=\left[\left(p_{1} q_{1} P-c_{1}\right)+\left(p_{2} q_{2} M-c_{2}\right)\right] I=$ $\Pi_{1}+\Pi_{2}$, where $\Pi_{1}=\left(p_{1} q_{1} P-c_{1}\right) I$ and $\Pi_{2}=\left(p_{2} q_{2} M-c_{2}\right) I$ are the net economic revenue for immature forestry biomass $P$ and mature forestry biomass $M$, respectively. We have found the bionomic equilibrium $\left(P_{\infty}, M_{\infty}, I_{\infty},\right)$ from the following simultaneous equations:

$$
\begin{aligned}
& (r+\gamma) P-\frac{r P^{2}}{K}-\beta P\left(t-h_{1}\right)-q_{1} P I-d_{1} P I=0, \\
& \beta P\left(t-h_{1}\right)-q_{2} M I-d_{2} M I-\mu M=0, \\
& {\left[\left(p_{1} q_{1} P-c_{1}\right)+\left(p_{2} q_{2} M-c_{2}\right)\right] I=0 .}
\end{aligned}
$$

Here, we consider (70) as the zero profit line. Harvesting is profitable when revenue for both the populations is greater than the cost, i.e., $p_{1} q_{1} P>c_{1}$ and $p_{2} q_{2} M>c_{2}$. When harvesting continues in both the populations, then $P_{\infty}=\frac{c_{1}}{p_{1} q_{1}}$ and $M_{\infty}=\frac{c_{2}}{p_{2} q_{2}}$. From equation (69), we get $I_{\infty}=\frac{\beta c_{1} q_{2} p_{2}}{c_{2} p_{1} q_{1}}-\mu$. Hence bionomic equilibrium $\left(P_{\infty}, M_{\infty}, I_{\infty}\right)$ exists when the inequality $\frac{\beta c_{1} q_{2} p_{2}}{c_{2} p_{1} q_{1}}>\mu$ holds. 


\section{Quantitative analysis and simulation}

To show the feasibility of the analytic results described in Section 4, we present the numerical results using MATLAB and Mathematica software. To verify the results, we consider three parametric sets as follows:

Set 1: $r=0.4, k=70, \beta=0.7, a_{1}=0.3, a_{2}=0.26, c_{1}=0.01, c_{2}=0.02, \tau_{1}=0.25, \tau_{2}=0.2$, $q_{1}=0.03, q_{2}=0.025, d_{1}=0.1, d_{2}=0.12, p_{1}=0.35, p_{2}=0.3, \gamma=0.3, \mu=0.01, d_{3}=0.12$;

Set 2: $r=1.2, k=70, \beta=0.7, a_{1}=0.3, a_{2}=0.26, c_{1}=0.01, c_{2}=0.02, \tau_{1}=0.25, \tau_{2}=0.2$, $q_{1}=0.03, q_{2}=0.025, d_{1}=0.01, d_{2}=0.012, p_{1}=0.35, p_{2}=0.3, \gamma=0.3, \mu=0.2, d_{3}=0.42$;

Set 3: $r=1.2, k=70, \beta=0.7, a_{1}=0.3, a_{2}=0.26, c_{1}=0.01, c_{2}=0.02, \tau_{1}=0.25, \tau_{2}=0.2$, $q_{1}=0.03, q_{2}=0.025, d_{1}=0.1, d_{2}=0.12, p_{1}=0.35, p_{2}=0.3, \gamma=0.3, \mu=0.01, d_{3}=0.12$.

Using set 1 , the trivial equilibrium case (extinction of all the population) is shown in Figure 2. For set 2, the axial equilibrium case $\tilde{E}$ (extinction of industrial density) is shown in Figure 3 with numerically calculated values $(46.664,163.337,0)$, and using set 3 the interior equilibrium case $\hat{E}$ (all the population coexist) is shown in Figure 4 with $(6.296,5.637$, 5.324) as the numerical values for $\hat{P}, \hat{M}$, and $\hat{I}$. 3d-plot for the stable graph when $h_{1}=0.72$ is shown in Figure 5. As we introduce delay, the system becomes unstable as it crosses the critical value $h_{1}=0.76$; the time series graph and $3 \mathrm{~d}$-plot for the same are shown in Figures 6 and 7, respectively. Also, for set 3, we get $b_{1}(0)=-0.1602-16.10 i, \mu_{2}=0.1654$, $m_{2}=-0.3458$, and $T_{2}=2.001$. Since $\mu_{2}>0$, the Hopf bifurcation is supercritical and the direction of the bifurcation $h>h_{10}$. Also $m_{2}<0$ and $T_{2}>0$, this implies that the bifurcating periodic solutions arising from $\hat{E}$ at $h_{10}$ are stable and the periods of limit cycle increase. Graphical representation of increasing legal and illegal logging $d_{1}, d_{2}$ and their effect on immature and mature forestry biomass (both populations decrease) is shown in Figures 8 and 9. Figure 10 depicts the decreasing label of industries on increasing the non-industrial uses of mature forestry biomass.

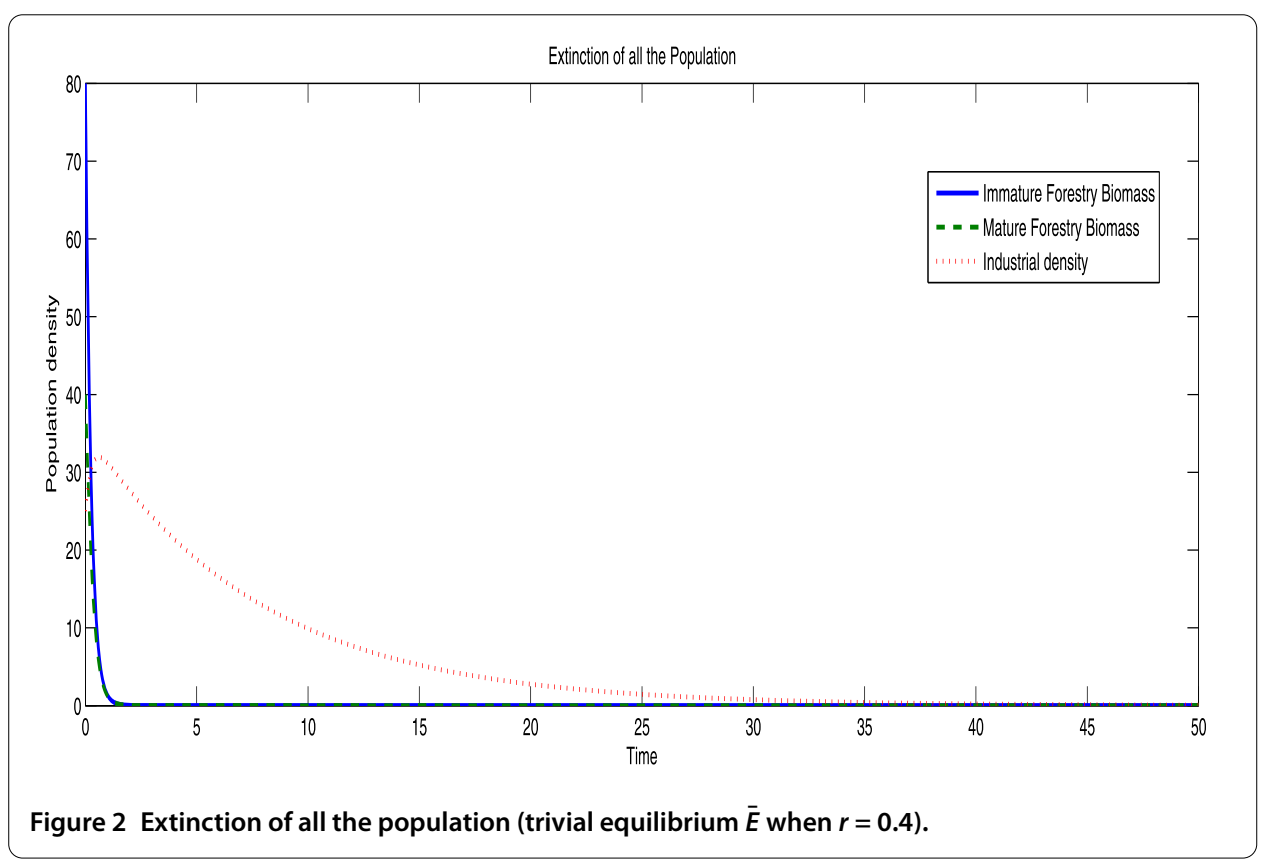



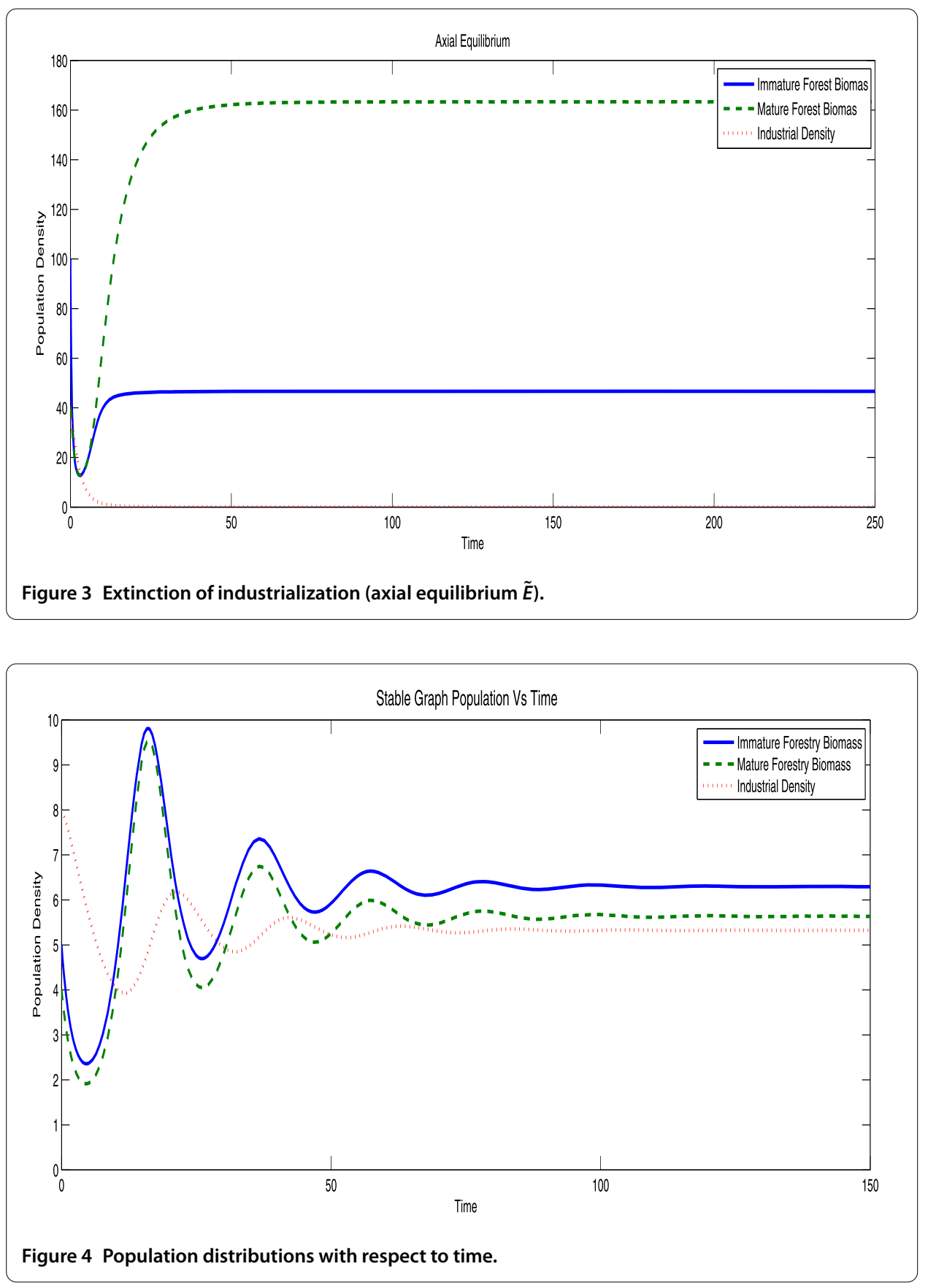

\section{Optimal harvesting policy}

In this section, our objective is to maximize the present value of a continuous time stream of revenue given by

$$
Q=\int_{0}^{\infty} e^{-\delta t}\left\{\left(p_{1} q_{1} P-c_{1}\right) I+\left(p_{2} q_{2} M-c_{2}\right) I\right\} d t
$$

where $\delta$ is continuous discount rate for the harvesting of forestry biomass. Here, our objective is to maximize the present value of $\mathrm{Q}$ subject to the system equations (1)-(3) and control constraints $\left(\tau_{i}\right)_{\min } \leq \tau \leq\left(\tau_{i}\right)_{\max }(i=1,2)$. Hamiltonian for the above control prob- 

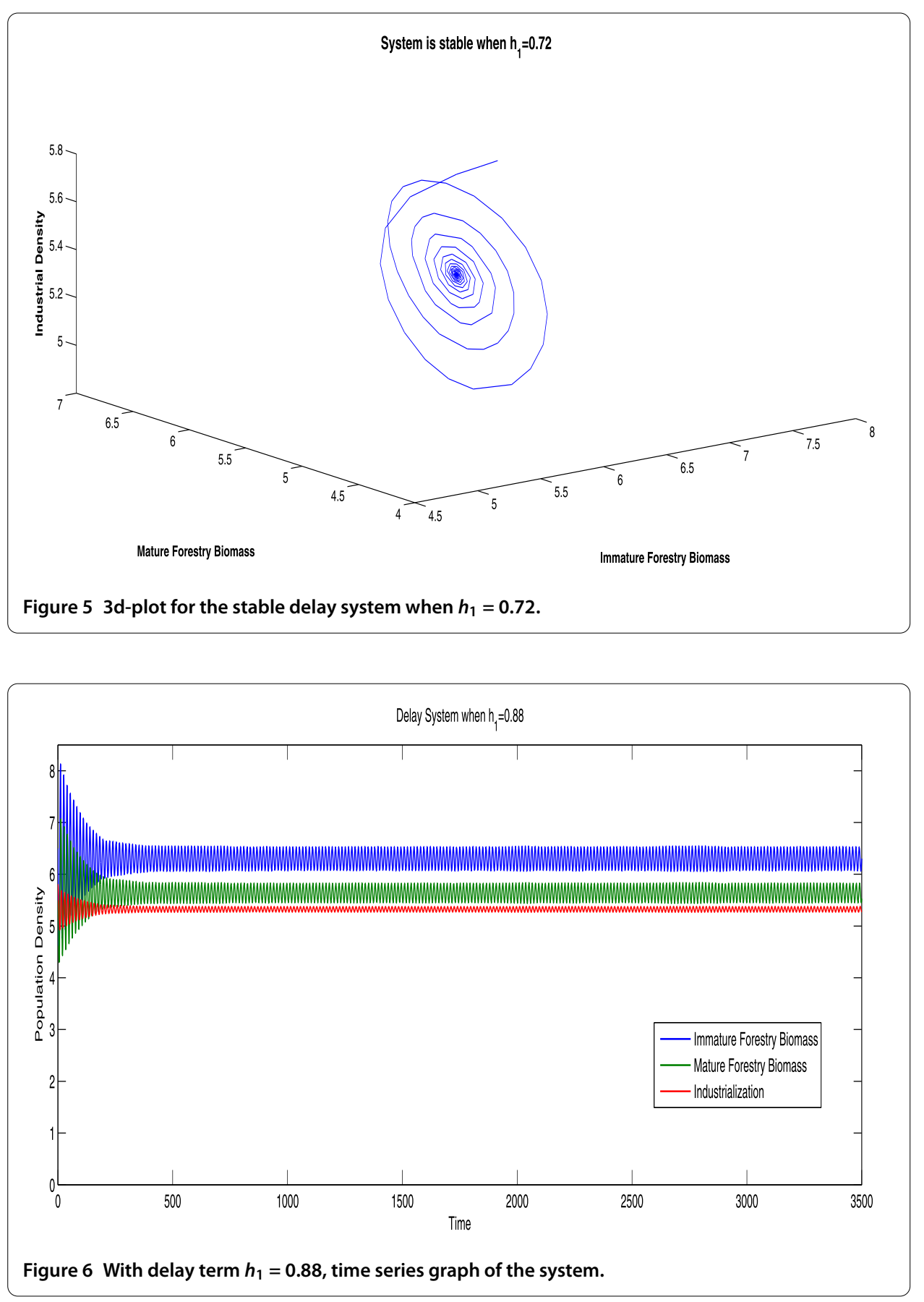

lem is defined as follows:

$$
\begin{aligned}
H= & e^{-\delta t}\left[p_{1} q_{1} P-c_{1}+p_{2} q_{2} M-c_{2}\right] I \\
& +\lambda_{1}(t)\left[(r+\gamma) P-\frac{r P^{2}}{K}-\beta P\left(t-h_{1}\right)-q_{1} P I-d_{1} P I\right] \\
& +\lambda_{2}(t)\left[\beta P\left(t-h_{1}\right)-q_{2} M I-d_{2} M I-\mu M\right]+\lambda_{3}(t)\left[a_{1}\left(\left(p_{1}-\tau_{1}\right) q_{1}+p_{1} d_{1}\right) P\right. \\
& \left.+a_{2}\left(\left(p_{2}-\tau_{2}\right) q_{2}+p_{2} d_{2}\right) M-a_{1} c_{1}-a_{2} c_{2}-d_{3} I\right]
\end{aligned}
$$



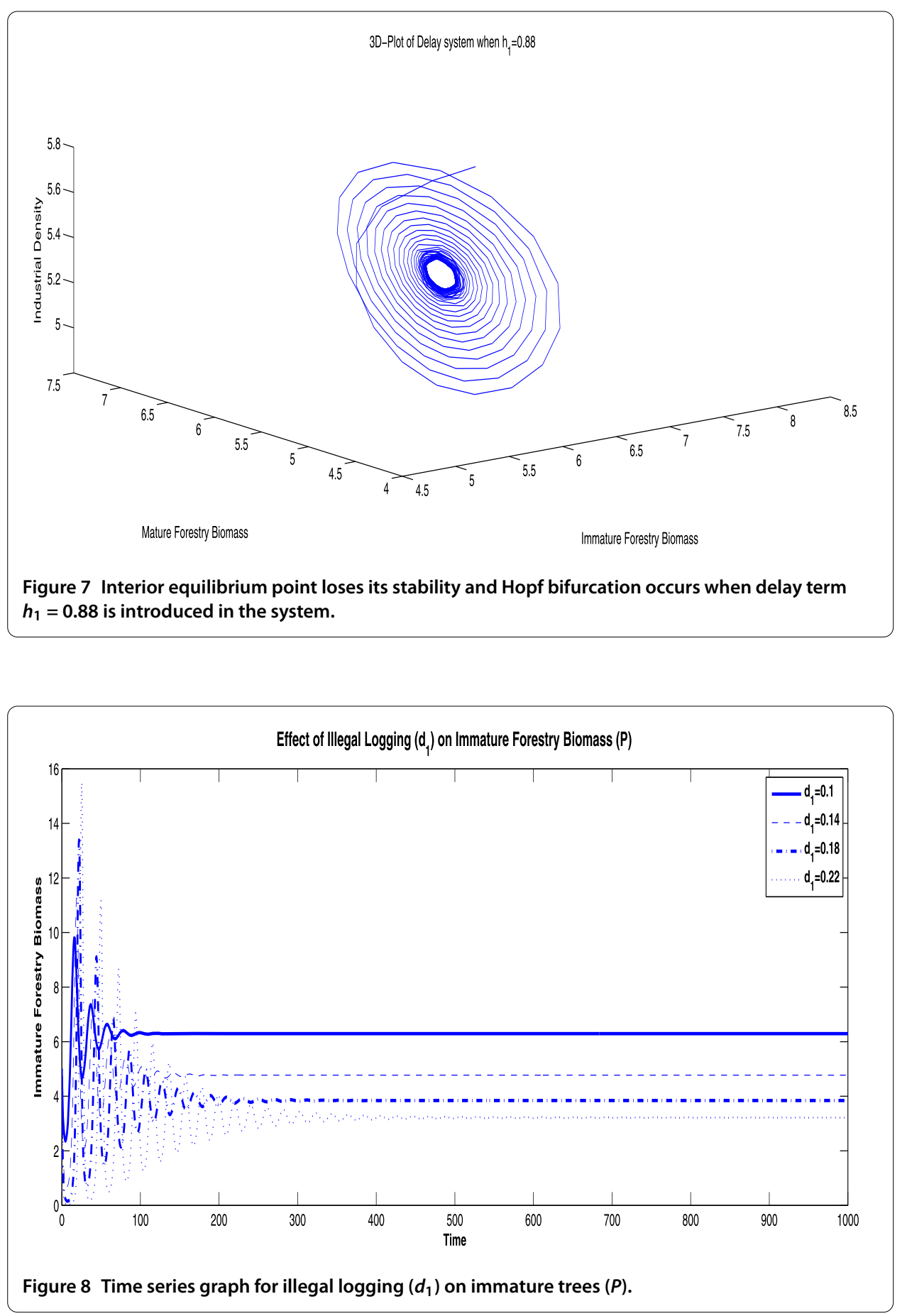

where $\lambda_{1}(t), \lambda_{2}(t)$, and $\lambda_{3}(t)$ are the adjoint variables. For $H$ to be maximum on the control set $\left(\tau_{i}\right)_{\min } \leq \tau \leq\left(\tau_{i}\right)_{\max }$ for $i=1,2$, we must have $\frac{\partial H}{\partial \tau_{i}}=0$, i.e., $\lambda_{3}=0$. This gives a necessary condition for a singular control to be optimal. For the optimal control, using Pontryagin's principle [20, 21], model equations (1)-(3), and the interior equilibrium, we can write

$$
\frac{d \lambda_{1}}{d t}=-e^{-\delta t} p_{1} q_{1} \hat{I}+\frac{r \hat{P}}{K} \lambda_{1}-\beta \lambda_{2}
$$



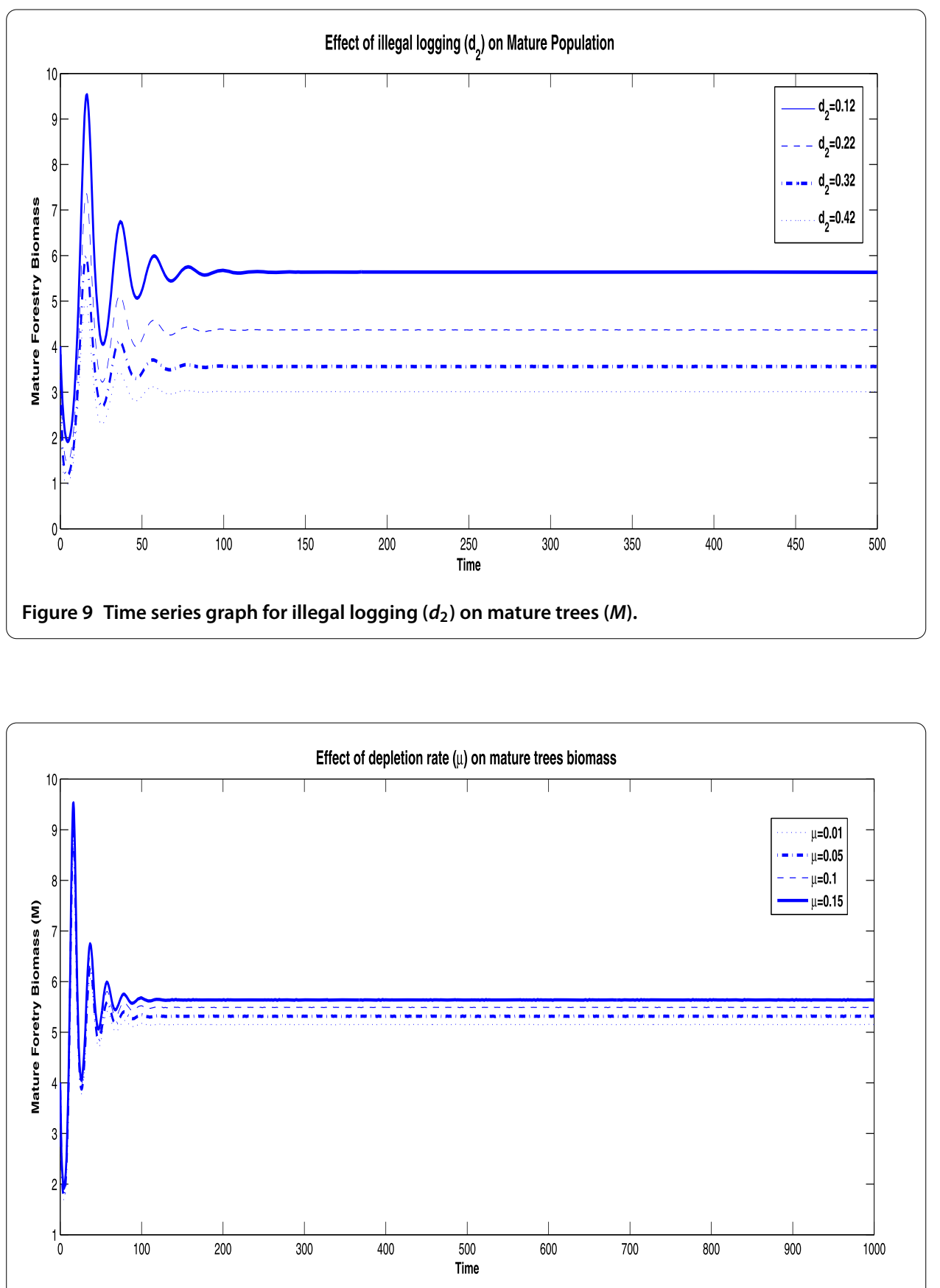

Figure 10 Depletion rate $(\mu)$ on mature trees $(M)$.

$$
\begin{aligned}
& \frac{d \lambda_{2}}{d t}=-e^{-\delta t} p_{2} q_{2} \hat{I}+\frac{\beta \hat{P}}{\hat{M}} \lambda_{2}, \\
& \frac{d \lambda_{3}}{d t}=-e^{-\delta t}\left(p_{1} q_{1} \hat{P}+p_{2} q_{2} \hat{M}-c_{1}-c_{2}\right)-\left(q_{1}+d_{1}\right) \hat{P} \lambda_{1}-\left(q_{2}+d_{2}\right) \hat{M} \lambda_{2}=0 .
\end{aligned}
$$

Solving equations (72), (73), we have $\lambda_{2}=-\frac{s_{1}}{s_{2}+\delta} e^{-\delta t}+c_{1} e^{s_{2} \delta}$ and $\lambda_{1}=-\frac{s_{3}}{s_{4}+\delta} e^{-\delta t}+c_{1} e^{s_{4} \delta}$, where $s_{1}=p_{2} q_{2} \hat{I}, s_{2}=\frac{\beta \hat{P}}{\hat{M}}, s_{3}=\frac{r \hat{P}}{K}$, and $s_{4}=-p 1 q_{1} \hat{I}+\frac{\beta_{1} s_{1}}{s_{2}+\delta}$. The values of $\lambda_{1}$ and $\lambda_{2}$ are 
bounded as $t \rightarrow \infty$. Therefore, we can write $\lambda_{1}$ and $\lambda_{2}$ as

$$
\lambda_{2}=-\frac{s_{1}}{s_{2}+\delta} e^{-\delta t}
$$

and

$$
\lambda_{1}=-\frac{s_{3}}{s_{4}+\delta} e^{-\delta t}
$$

On substituting the values of $\lambda_{1}$ and $\lambda_{2}$ in equation (74), we get the optimal singular path for the optimum equilibrium level as

$$
\begin{aligned}
& \left(s_{4}+\delta\right)\left(s_{2}+\delta\right)\left[c_{1}+c_{2}-p_{1} q_{1} \hat{P}-p_{2} q_{2} \hat{M}\right] \\
& \quad-\left(s_{2}+\delta\right)\left(d_{1}+q_{1}\right) \hat{P} s_{3}-s_{1}\left(s_{4}+\delta\right)\left(d_{2}+q_{2}\right) \hat{M}=0
\end{aligned}
$$

where $\hat{P}=P_{\delta}, \hat{M}=M_{\delta}$, and $\hat{I}=I_{\delta}$ are the optimum levels of population densities. Also, from (75) and (77), we can calculate $\lambda_{1}$, which in this case is defined as

$$
\lambda_{1}=\frac{\left(-p_{1} q_{1} \hat{P}+p_{2} q_{2} \hat{M}-c_{1}-c_{2}\right)\left(s_{2}+\delta\right)+s_{1} \hat{M}\left(q_{2}+d_{2}\right)}{e^{\delta t}\left(q_{1}+d_{1}\right) \hat{P}} .
$$

On comparing (75)-(78), we have

$$
-\frac{s_{3}}{s_{4}+\delta} e^{-\delta t}=\frac{\left(-p_{1} q_{1} \hat{P}+p_{2} q_{2} \hat{M}-c_{1}-c_{2}\right)\left(s_{2}+\delta\right) e^{-\delta t}+s_{1} \hat{M}\left(q_{2}+d_{2}\right)}{\left(q_{1}+d_{1}\right) \hat{P}}
$$

Hence, solving the steady state equations together with (79), we get the optimal tax and optimal solution $\hat{P}=P_{\delta}, \hat{M}=M_{\delta}$, and $\hat{I}=I_{\delta}$, which could be applied to harvest forestry biomass in a conservative way.

\section{Sensitivity analysis}

The relative change in the state variable when the respective parameter changes is the sensitivity analysis. The sensitivity of interior equilibrium $\hat{E}$ with corresponding parameters is analyzed using the normalized forward sensitivity index, and the sensitivity of parameters is shown using the bar graph (see Figure 11). From the bar graph in Figure 11, we notice the following:

1. Parameters $\beta, a_{1}, p_{1}$ are negatively sensitive, and $r, K, a_{2}, p_{2}, \gamma$ are positively sensitive parameters to immature forestry biomass $(\hat{P})$.

2. Parameters $r, a_{2}, d_{2}, p_{2}$ are negatively sensitive, and $K, \beta, d_{1}, p_{1}, \gamma$ are positively sensitive ones to mature forestry biomass $(\hat{M})$.

3. Parameters $a_{1}, q_{1}, d_{1}, p_{1}$ are negatively sensitive, and $r, a_{2}, p_{2}$ are positively sensitive parameters to industrial density $(\hat{I})$.

\section{Result and discussion}

The dynamical mathematical approach makes it possible to build up the forestry model to accommodate disturbances arising in the presence of excess illegal logging. An important consideration in the study of forestry and industrial density-based dynamical delay model 


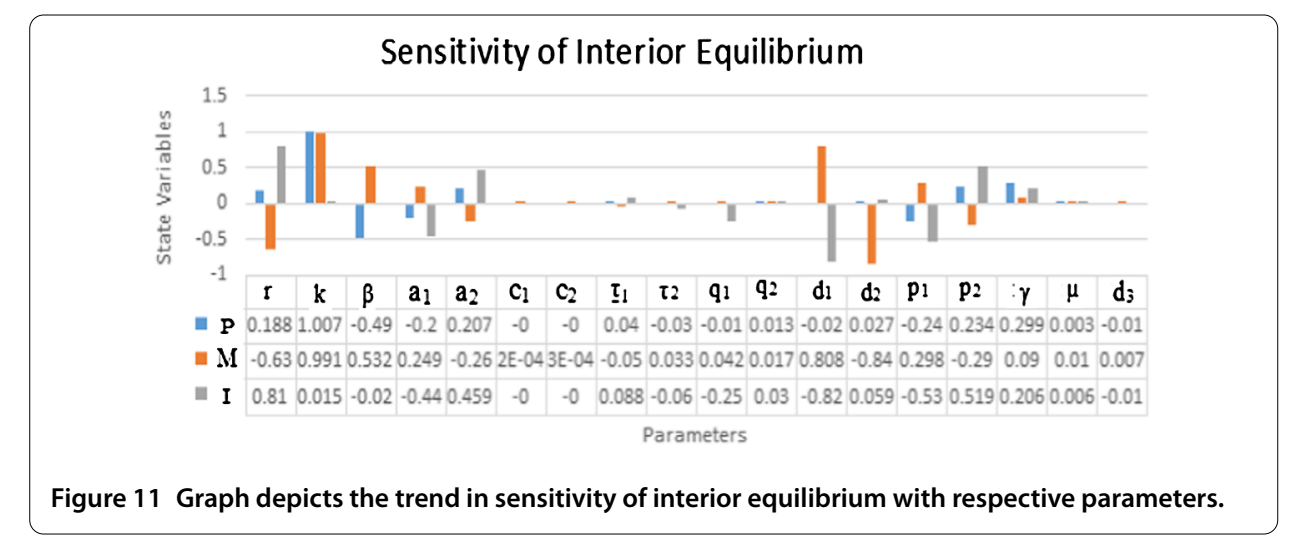

is the role of illegal logging and the depletion rate of mature trees for non-industrial uses. For the present system, we have considered $\beta$ as the transition rate of immature to mature population and $h_{1}$ as the time taken to convert the immature population to mature population, which plays a crucial role in the model formulation. In general bifurcation theory is concerned with the dynamical system which contains one or more external parameters and with the manner in which the solution set may undergo structural changes as the parameter or parameters are varied. In present problem, $h_{1}$ is that external parameter. When the delay parameter is greater than the critical threshold $h_{1}=0.72$, the model starts bifurcating. In this study, it is observed that in the absence of maturation delay, the system is locally asymptotically stable, while in the presence of maturation delay, a Hopf bifurcation is observed as the delay parameter $\left(h_{10}\right)$ reaches the critical threshold. As illegal logging increases in forestry, both immature and mature forestry biomass decrease. Numerically, using parametric set 3 , the value of $h_{1}$ is 0.78 . The trivial, axial, and coexisting states are graphically presented using set 1,2 , and 3 , respectively. Optimal control policy has been discussed by constructing the Hamiltonian which is further analyzed using Pontryagin's principle. We have observed that in the case of infinite discount rate economic rent tends to zero. We have found the bionomic equilibrium and also obtained the optimal path for the optimal equilibrium level of forestry biomass and industrial density. We have found the sensitivity of the coexisting state using the normalized forward sensitivity index using the parameters mentioned in set 3 . The sensitivity of parameters depends upon the choice of parameters. From the analysis of this tool, we have observed that if we consider small (or large) values of the parameters then, accordingly, the outcome is highly negatively (or positively) sensitive.

\section{Acknowledgements}

The first author is thankful to Prof. Joydip Dhar and Prof. O.P. Misra and the Department of Science and Technology, Government of India for the financial support vide reference No. SR/WOS-A/MS-21/2013 under Women Scientist Scheme to successfully carry out this research work. The second author thankfully acknowledges the National Board of Higher Mathematics in form of PDF with Ref. No. (2/40(29)/2014/R\&D-II) for the financial support.

\section{Competing interests}

The authors declare that they have no competing interests.

Authors' contributions

All authors read and approved the final manuscript.

\section{Author details}

1 'Department of Applied Sciences, Indian Institute of Information Technology and Management, Morena Link Road, Gwalior, Madhya Pradesh 474015, India. ²Department of Mathematics and Astronomy, Lucknow University, Lucknow, U.P., India. 


\section{Publisher's Note}

Springer Nature remains neutral with regard to jurisdictional claims in published maps and institutional affiliations.

Received: 2 August 2017 Accepted: 6 December 2017 Published online: 19 December 2017

\section{References}

1. Bussmann, RW: Destruction and management of Mount Kenya's forests. Ambio 25(5), 314-317 (1996)

2. Doornbosch, R, Steenblik, R: Round table on sustainable development, biofuels: is the cure worse than the disease (2007)

3. Lawson, S, MacFaul, L: Illegal logging and related trade: indicators of the global response (2010)

4. Carle, J, Holmgren, P: Wood from planted forests: a global outlook 2005-2030. For. Prod. J. 58(12), 6-18 (2008)

5. Hritonenko, N, Yatsenko, Y, Goetz, RU, Xabadia, A: Optimal harvesting in forestry: steady-state analysis and climate change impact. J. Biol. Dyn. 7(1), 41-58 (2013)

6. Khaine, I, Woo, SY: An overview of interrelationship between climate change and forests. For. Sci. Technol. 11(1), 11-18 (2015)

7. Misra, AK, Verma, M: Modeling the control of atmospheric carbon dioxide through reforestation: effect of time delay. Model. Earth Syst. Environ. 1(3), 1-17 (2015)

8. Chaudhary, M, Dhar, J, Misra, OP: Analysis of exploitation of forestry biomass by industrialization: effect of harvesting and taxation. Int. J. Comput. Appl. Math. 3, 311-325 (2017). https://doi.org/10.1007/s40819-017-0357-x

9. Banitalebi, A, Aziz, MIA, Aziz, ZA, Nasir, N: Modelling and optimization for palm oil plantation management. AIP Conf. Proc. 1750(1), Article ID 030046 (2016)

10. Chaudhary, M, Dhar, J: Forestry biomass conservation with synthetic industry: a mathematical model. In: 2013 Nirma University International Conference on Engineering (NUiCONE), pp. 1-5. IEEE, New York (2013)

11. Chaudhary, M, Dhar, J, Sahu, GP: Mathematical model of depletion of forestry resource: effect of synthetic based industries. Internat. J. Math. Comput. Phys. Electr. Comput. Eng. 7(4), 1-5 (2013)

12. Chaudhary, M, Dhar, J, Misra, OP: A mathematical model for the conservation of forestry biomass with an alternative resource for industrialization: a modified Leslie Gower interaction. Model. Earth Syst. Environ. 1(4), 1-10 (2015)

13. Chaudhary, M, Dhar, J, Misra, OP: Conservation of Forestry Biomass Introducing Variable Taxation for Harvesting: A Mathematical Model. World Scientific, Singapore (2016)

14. Kuang, Y: Delay Differential Equations: With Applications in Population Dynamics, vol. 191. Academic Press, San Diego (1993)

15. Birkhoff, G, Rota, GC: Ordinary Differential Equations (1978)

16. Celik, C: The stability and Hopf bifurcation for a predator-prey system with time delay. Chaos Solitons Fractals 37(1), $87-99$ (2008)

17. Misra, Ak, Lata, K: Modeling the effect of time delay on the conservation of forestry biomass. Chaos Solitons Fractals 46, 1-11 (2013)

18. Freedman, HI, Rao, VSH: The trade-off between mutual interference and time lags in predator-prey systems. Bull. Math. Biol. 45(6), 991-1004 (1983)

19. Hassard, BD, Kzrinoff, ND, Wan, WH: Theory and Application of Hopf Bifurcation. London Mathematics Society Lecture Note Series, vol. 41. Cambridge University Press, Cambridge (1981)

20. Clark, CW: Mathematical Bioeconomics: The Mathematics of Conservation, vol. 91. Wiley, New York (2010)

21. Pontryagin, LS: Mathematical Theory of Optimal Processes. CRC Press, Boca Raton (1987)

\section{Submit your manuscript to a SpringerOpen ${ }^{\circ}$ journal and benefit from:}

- Convenient online submission

- Rigorous peer review

- Open access: articles freely available online

- High visibility within the field

- Retaining the copyright to your article

Submit your next manuscript at $\gg$ springeropen.com 